Document downloaded from:

http://hdl.handle.net/10251/51095

This paper must be cited as:

Albert Gil, FE.; Gomis Martí, JM.; Blasco, J.; Valiente González, JM.; Aleixos Borrás, MN. (2015). A new method to analyse mosaics based on Symmetry Group theory applied to Islamic Geometric Patterns. Computer Vision and Image Understanding. 130:54-70. doi:10.1016/j.cviu.2014.09.002.

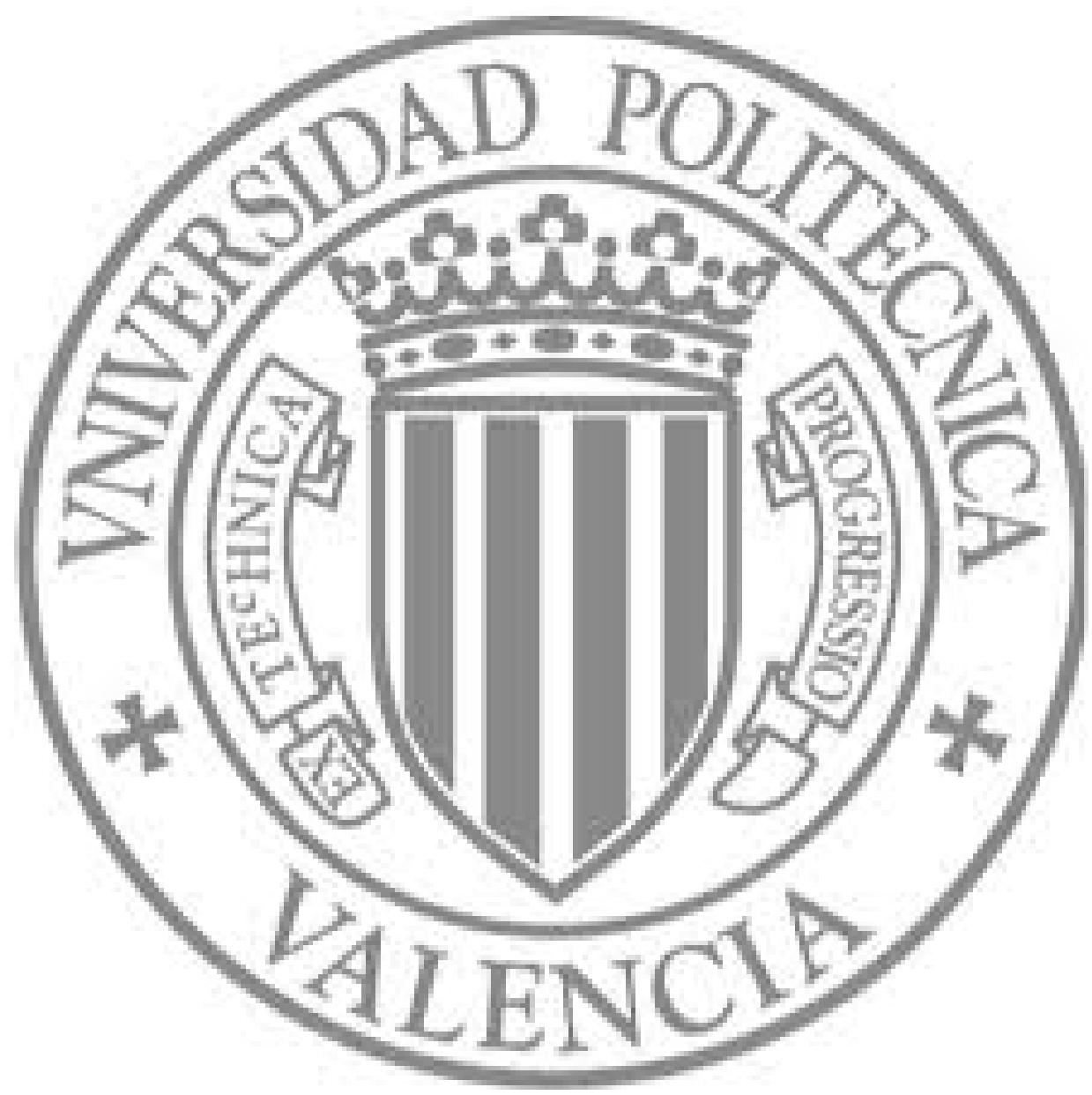

The final publication is available at

http://dx.doi.org/10.1016/j.cviu.2014.09.002

Copyright Elsevier 


\title{
A new method to analyse mosaics based on Symmetry Group theory applied to Islamic Geometric Patterns
}

\author{
F. Albert ${ }^{\mathrm{a}}$, J.M. Gómis ${ }^{\mathrm{a}}$, J. Blasco ${ }^{\mathrm{b}}$, J.M. Valiente ${ }^{\mathrm{c}}$, N. Aleixos ${ }^{\mathrm{a}}{ }^{*}$ \\ ${ }^{a}$ Inter-University Research Institute for Bioengineering and Human Centered Technology, Universitat \\ Politècnica de València, 46022-Valencia, Spain, \{fraalgi1, jmgomis, naleixos\} @dig.upv.es \\ ${ }^{\mathrm{b}}$ Instituto Valenciano de Investigaciones Agrarias - IVIA, 46113-Moncada, Valencia, Spain, \\ blasco_josiva@gva.es \\ ${ }^{c}$ Computer Engineering Department, Universitat Politècnica de València, 46022-Valencia, Spain, \\ jvalient@disca.upv.es
}

\begin{abstract}
This article presents a new method for analysing mosaics based on the mathematical principles of Symmetry Groups. This method has been developed to get the understanding present in patterns by extracting the objects that form them, their lattice, and the Wallpaper Group. The main novelty of this method resides in the creation of a higher level of knowledge based on objects, which makes it possible to classify the objects, to extract their main features (Point Group, principal axes, etc.), and the relationships between them. In order to validate the method, several tests were carried out on a set of Islamic Geometric Patterns from different sources, for which the Wallpaper Group has been successfully obtained in $85 \%$ of the cases. This method can be applied to any kind of pattern that presents a Wallpaper Group. Possible applications of this computational method include pattern classification, cataloguing of ceramic coatings, creating databases of decorative patterns, creating pattern designs, pattern comparison between different cultures, tile cataloguing, and so on.
\end{abstract}

Index Terms - Mosaics, Symmetry Groups, Wallpaper Groups, Point Groups, image understanding, tiles, Islamic Geometric Patterns, pattern classification.

\section{Introduction}

This paper proposes a method to get the understanding generally present in any kind of pattern that presents a Wallpaper Group (WG), which describes the arrangement of a set of objects repeated from a set of isometries. Unlike other existing works, which only identify the WG (the so-called "structure"), this method obtains the objects present in the pattern, allowing them to be classified and their symmetries obtained. Moreover, the information extracted from the objects is used to obtain the isometries that constitute the WG. An important contribution is the possibility of approaching the pattern analysis in two

\footnotetext{
* Corresponding author:

Tel.: + 34963879514

Fax.: +34963877419
} 
ways: by discarding the colour of each object as a discriminating feature, which allows a different WG to be obtained when there are objects with the same shape and different colour, and second by obtaining the WG of each object class, apart from the WG of the entire pattern, thus obtaining all the WG present in the pattern. This method has been implemented through an application, using the Image Processing and Pattern Recognition techniques and the mathematical principles of Symmetry Groups, and has been tested and validated on Islamic Geometric Patterns (IGP) and a number of alternative datasets (mainly from http://en.wikipedia.org/wiki/Wallpaper_group, and other paving slabs and fabrics).

\subsection{Definitions}

The main terms used in this article are defined as follows:

- Region: set of neighbouring pixels in the image with similar colour, which are perceived as a unit.

- Object: geometric figure matching a region with a closed contour and a series of properties (colour, area, perimeter, etc.).

- Shape: description of the object depending on the points or parts making up the outer contour and how they are interrelated in terms of positions, orientations and relative sizes [1]. Shape is the main feature of an object and in this work shape and contour are used interchangeably.

- Object class: set of objects with the same shape, size and colour.

- Isometries: geometric transformations which, when applied to an object, do not change its shape. The isometries are: translations, rotations and reflections (symmetry axes). A glide reflection is the combination of a reflection relative to a symmetry axis and a translation in the direction of the same axis.

- Pattern: a repeated set of elements. In this work the elements are the objects, and they are duplicated and combined by means of isometries without overlap, covering a flat surface.

- Primitive Cell (PC) or Unit Lattice: the minimum part of the pattern that can generate the whole pattern by making copies and displacements. The Primitive Cell may be an object or a set of objects.

- Piece or Tile: fragment of thin ceramic material, with the outer face glazed, used to cover floors and walls.

- Islamic Geometric Patterns (IGP) or Islamic Mosaics: basic decorative elements of Islamic architecture consisting of a pattern formed by a set of small pieces with simple shapes (straight lines and arcs). In this work, for the purposes of the image processing of IGPs, the pieces are considered as objects. 


\subsection{Symmetry Groups}

A Symmetry Group (SG) is the set of all isometries $\sigma$, which transforms a set of objects $S$ into itself $(\sigma S=S)$. There are four types of Symmetry Groups: Point Groups, Frieze Groups, Wallpaper Groups and Crystallographic Groups, depending on whether they have 0, 1, 2 or 3 independent translations [2] [3] [4]. This paper deals with Point Groups and Wallpaper Groups.

The Point Group (PG) describes the group of all isometries under which an object is invariant. The classes of PG are the following:

- Rotational symmetry (also called $n$-fold rotational symmetry or cyclic symmetry): cyclic groups $C_{1}, C_{2}, \ldots, C_{n}$, where $C_{n}$ (of order $n$ ) consists of all rotations about a fixed point (the centroid) by multiples of $360^{\circ} / n$ (Figure 1a Cyclic Group C3 with $120^{\circ}$ rotations).

- Reflection symmetry (also called mirror symmetry or dihedral symmetry): dihedral groups $D_{1}, D_{2}$, $\ldots, D_{n}$, where $D_{n}$ (of order $2 n$ ) consists of the rotations in $C_{n}$ together with reflections in $n$ symmetry axes that pass through the centroid (Figure 1b Dihedral Group D2 with $180^{\circ}$ rotation and two symmetry axes).

The WG describes the arrangement of a set of objects which are repeated to form a pattern, from a set of isometries that always include two translations. There are 17 different WG [3]. The two smallest independent translations in the pattern define the lattice. The PC is the smallest part of the pattern repeated by lattice translations. Figure 1 shows the output of the application developed for two different objects and two patterns. Figure 1c shows a WG CMM with glide and non-glide symmetry axes and $180^{\circ}$ rotations, and Figure $1 \mathrm{~d}$ shows a WG P3 with $120^{\circ}$ rotations and without any kind of symmetry axes. The two patterns are shown with the Primitive Cell.

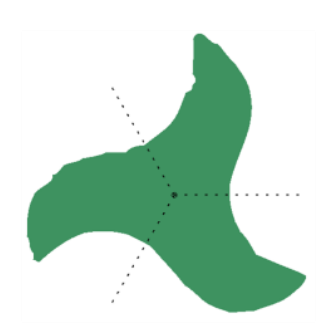

a)

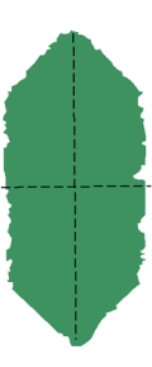

b)

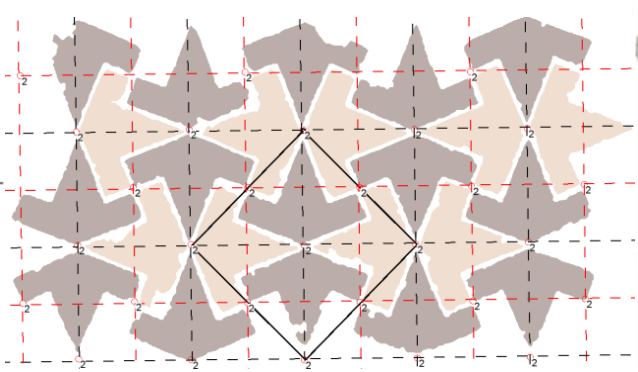

c)

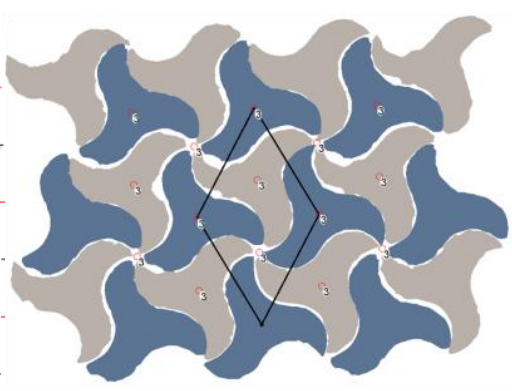

d)

Figure 1. Output of the application for some samples: a) Object with Point Group C3. The three sectors of the object appear separated with dotted lines; b) Object with Point Group D2. The symmetry axes are represented with dashed lines; c) and d) Patterns with WG. The solid line is the Primitive Cell. Symmetry axes are represented with dashed lines: red for glide and black for non-glide symmetry axes. Rotation 
centres are represented with small circles with a number (two for $180^{\circ}$ rotations and three for $120^{\circ}$ rotations)

\subsection{State of the art}

The scientific literature contains research studies that generate or analyse IGP based on SG, and some others based on different geometric approaches. Among the SG-based works, there are many examples of pattern generation [5] [6] and the development of computer-aided design allowed the creation of software for generating patterns [7] [8], but this is straightforward, since it just consists in geometric transformations applied repetitively. As for analysing IGP, special mention should be made of the theoretical approach by [9], who roughly described the steps to be followed in pattern analysis, i.e. identifying the PC and obtaining the WG. In [10], the authors presented an application which obtains the translations, symmetry axes and centres of rotation of an image via image correlations with itself translated, reflected or rotated, respectively, to determine the PC and WG using heuristic methods. This computer application is one of the most comprehensive contributions in the area of image analysis based on SG, although precisely due to its simplicity (it does not deal with objects), no information can be obtained beyond that deduced from just the colour of pixels. Instead of correlations, [11] extracted the lattice of patterns using Distance Matching Functions (DMFs). Much like previous works, [12] [13] also obtained the PC and the WG, but using Wavelets and a Nearest Neighbour classifier. In [14], the authors used Liu's method described in [10] to obtain the PC, and the WG in order to detect defects in patterns. As they pointed out, the WG cannot be obtained if the number of defects is relevant. On the other hand, [15] analysed IGP obtaining their Point Group (for rosettes), Frieze Groups (for friezes) or Wallpaper Groups, and in order to obtain the isometries of patterns of the image itself, correlations between translations, reflections or rotations were performed. The final description of an IGP was a feature vector including the Symmetry Group of the pattern and a colour histogram of the Primitive Cell (patterns with Frieze or Wallpaper Groups) or Fold (patterns with Point Group) but did not consider objects, the only contributions being the use of rosettes, friezes and the colour histogram.

With respect to the non SG-based works, there are many different methods for generating patterns, for example from rosettes [16] or from two of the most common pieces used in IGP called Zohreh and Sili [17]. A review of the different methods can be found in [18]. For analysing Islamic Geometric Patterns [19] proposed a theoretical method based on two features that were defined as Minimum Number of Grids (MNG) and Lowest Geometric Shape (LGS). [20] also proposed a method for analysing Islamic Patterns, which presented a Point Group (rosettes), based on the decomposition of a square image into a quadtree structure searching for symmetries at different levels of abstraction; this method resembled the work of the 
ancient artisans consisting in drawing the motif on a folded paper and then unfolding it.

Not all the research papers cited that present proposals for the classification of IGP come with software tools, and those that do include them only operate at pixel level, but do not retrieve the objects that form the pattern. Therefore, it is impossible to obtain their symmetries, and image cataloguing is performed using only colour information. Hence, they are not able to find alternative WG in patterns when there are identical objects that differ in colour, which frequently occurs. In fact, in [19], the authors criticised the methods based on Symmetry Groups because they did not reflect the way of thinking of the artisans (how to combine the pieces) and were a rather general approximation that failed to explore edition capabilities (to generate new patterns). Such criticism can be applied to the methods proposed in [10] [11] [12].

Thus, in this paper we propose a new method for analysing images of Islamic Geometric Patterns, and its key feature is the incorporation of a higher level of abstraction: the objects forming the pattern. This method has led to the development of an application. The information found can be used to generate new patterns. Earlier versions of the method have been reported in [21] [22] [23]. The main contributions of this research are the following:

- Pattern analysis is more comprehensive: the objects are obtained and classified, and their Point Group is also obtained.

- Obtaining the isometries, and therefore the WG, not only from the colours of the pixels, but also from features extracted from the objects (considering colour, shape, Point Group and orientation). Thus, the colour can be discarded when classifying objects, so if there are objects with the same shape and different colours, patterns with more than one WG can be found.

- The WG is obtained for each class of objects. So, if there are objects with different WG, they can be found.

The paper is organised as follows. In section 2 a brief description of the proposed method is presented. Sections 3 to 5 offer a detailed description of the different levels of the method: Image Level, Object Level and Structure Level. Section 6 presents the results of the application of the proposed method on a set of IGP samples, the parameters of each stage and the computational cost, and the results on other sets containing Wikipedia WG samples and some images of paving slabs from public constructions and fabrics, in order to show the flexibility of the method. Finally, section 7 reports the conclusions and further work.

\section{Description of the Method}

The method is based on stages according to three levels of knowledge that they include: a) Image or pixel-oriented level, in which the image is segmented to obtain the different regions of interest; b) Objectoriented level, in which the regions obtained correspond to objects and their features extracted including 
the contour and Point Group; and c) Structure Level, in which the translations, rotations and symmetry axes existing in the pattern, that is, the structure or Wallpaper Group, are found. Figure 2 shows the scheme of the methodology proposed. All the process that supports this methodology is entirely automatic, except the tuning of the parameters used and the configuration of the Image Level (i.e. the segmentation method applied in 3.2).

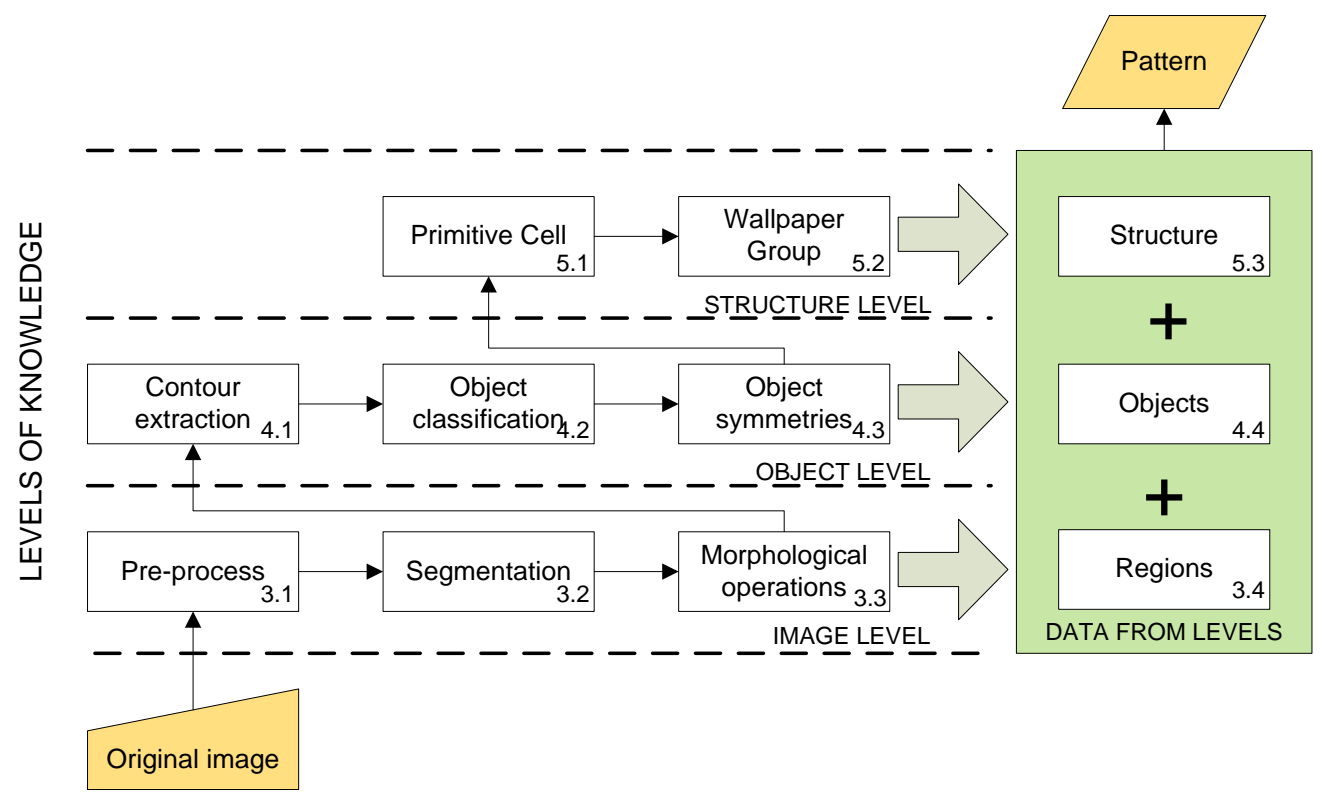

Figure 2. Scheme of the method including the stages and information obtained for each level. The numbers of the sections where the stages are described are stated at the bottom right of each box

The initial values of the parameters have been obtained using an implementation of the optimization algorithm Simulated Annealing described in [24], using a set of patterns with different Wallpaper Groups to determine a set of optimal values for the parameters. The final adjustments to obtain successfully the complete results (all classes of objects with their Point Group) require in some specific cases fine manual adjustment after outcome. The computational cost, the parameters used and the intermediate graphical results of the algorithm at each stage are described in section 6.2.

\section{Image Level}

This level is a pixel-oriented level, and its purpose is to extract the different regions of interest in the image, which is achieved by means of pre-processing, segmentation and morphological operations.

\subsection{Pre-processing}

A pre-processing stage is performed to reduce noise present in the image. This stage is performed using two different low-pass filters: Gaussian blur or Median. 


\subsection{Segmentation}

The next stages of the application depend greatly on the quality of the segmentation. A wide variety of image segmentation approaches have been reported in the literature [25] [26] [27].

Two different ways to segment the images have been implemented in this work. The first is through a clustering technique based on the Mean-Shift algorithm [28] [29], which is appropriate when the edges between regions are well defined. The second method consists in an edge-based technique, like the Watershed algorithm [30], which is more appropriate for images with weak edges between the regions because it emphasises the edges.

\subsection{Morphological operations}

Later, operations like Erosion, Dilation, Opening and Closing have been applied in order to remove small regions and holes or to separate linked regions [31], so the remaining regions will be considered the objects of the image. Finally, Hu moments [32] were used to calculate the properties of the objects like area, centroid, angle and size of principal axes that will be used in the following stages.

\subsection{Description of regions}

The result after applying the operations included in the Image Level consists of $m$ regions. At the end of this level, an object $O_{k}$ contains only the properties of its corresponding region: $O_{k}, k \in[0, m-1]$ is described using a tuple $O_{k}=\left(R^{k}\right.$, colour $\left.^{k}, p^{k}, A^{k}, \varphi_{\text {min }}^{k}, I_{\text {min }}^{k}, I_{\text {max }}^{k}\right)$, where:

- $R^{k}=\left\{p_{i}\right\}, i \in[0, n-1]$ : set of $n$ pixels belonging to the region.

- colour $^{k}$ : average colour of pixels of $R^{k}$.

- $p_{c}^{k}=\left(x_{c}, y_{c}\right)$ : centroid of the region.

- $A^{k}$ : area (number of pixels of $R^{k}$ ).

- $\varphi_{\text {min }}^{k}$ : angle of major axis.

- $I_{\text {min }}^{k}, I_{\text {max }}^{k}$ length of principal axes.

\section{Object Level}

The goal of this level is to complete the objects by extracting the shape (contour) of the regions found on the previous level, to classify them, and to obtain the Point Group for each class of objects.

\subsection{Contour extraction}

In order to extract the contour of each region, a boundary-following algorithm [33] has been used, thus the contour $C$ is a closed sequence of 8-connected points defined as following: $C=\left\{p_{i}\right\}, i \in[0, m-1]$, where $p_{i}$ are 
the contour points and $m$ is the number of points of such a contour.

\subsection{Object classification}

Unlike industrially manufactured objects, in IGP, the objects found in the images are repeated with slight differences between them due to fact that they are handmade. These differences can affect both the shape and the orientation, and hence in order to obtain a classification of the objects these two features must be taken into account. In this article, the classification methodology is based on an all-against-all comparison process (each object is compared to all other objects) using a coarse-to-fine strategy (first a quick comparison to discard obvious cases and later a precise comparison to detect smaller differences) in order to optimise the computational cost. Firstly, each pair of objects is compared using the area and the length of the principal axes. If the two objects are geometrically similar in this coarse comparison, they are compared using a 'fine' process to obtain a measurement of their dissimilarity [34].

The works presented in [35] and [36] also perform a process of fine comparison using the Canny edge detection algorithm to extract objects and then to obtain features like relevant points, type of sides, relative lengths, orientation and concavity/convexity, colour information and also the topological relationships among them. Dissimilarity was calculated by comparing one-by-one the types of sides between the relevant points that were found, and also by using the relative information about size and orientation, in a similar way to how [37] compared contours looking for tangencies, concentricity, parallelism, etc. The problem with this technique is the noise typically present in the images of IGP, which makes it difficult to obtain all of these characteristics with accuracy.

To deal with this problem, in this work, the fine comparison has been performed by using the contourbased method described in [38]. In particular, by assuming the contour of the objects to be a discrete and periodic function $f[n]=\left(x_{n}, y_{n}\right), n \in[0, N-1]$ :

1. The contours were filtered by means of a discrete convolution using a 1D Gaussian filter.

2. The contours were re-sampled to 256 equidistant points because they must have the same number of points to be compared properly.

3. The coordinates of the points in each contour were converted so as to be relative to the centroid of its region.

Then, the dissimilarity $d\left(f_{1}, f_{2}\right)$ between the contours of two objects $O_{1}$ and $O_{2}$ was defined as:

$$
d\left(f_{1}, f_{2}\right)=\min _{\alpha, q} \sqrt{\frac{1}{N} \sum_{n=0}^{N-1}\left(f_{1}[n]-R_{\alpha} \cdot f_{2}[n+q]\right)^{2}}
$$

where $N$ is the number of points on the two contours (256 after resampling), $q$ is the starting point of 
contour $f_{2}$ to compare to $f_{1}, q \in[0, N-1]$, and $R_{\alpha}$ is a rotation of angle $\alpha$. Except in some cases, it is enough to consider the multiples of $30^{\circ}$ since in all cases the angles of rotation in Wallpaper Groups are $180^{\circ}, 120^{\circ}$, $90^{\circ}$ and $60^{\circ}$. The above expression is minimised with respect to $q$ (starting from any point) and $\alpha$ (orientation).

Since an object can also appear reflected in the image, the same process is repeated but taking the second contour reflected about the horizontal axis passing through the centroid of the object $f^{R}[n]=\left(x_{n},-y_{n}\right)$, $n \in[0, N-1]$, thus obtaining the dissimilarity of the reflected objects $d^{R}\left(f_{1}, f_{2}\right)=d\left(f_{1} f_{2}^{R}\right)$. Finally, those pairs of objects in the image are considered as belonging to the same class if $d\left(f_{1} f_{2}\right)<u$ or $d^{R}\left(f_{1} f_{2}\right)<u$, where $u$ is a user-chosen threshold in the range [0.1..1]. If $d^{R}\left(f_{1} f_{2}\right)<d\left(f_{1} f_{2}\right)$, the objects are reflected. See Figure 3 .
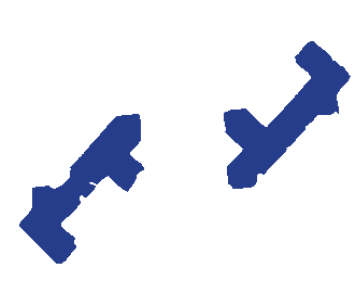

a)

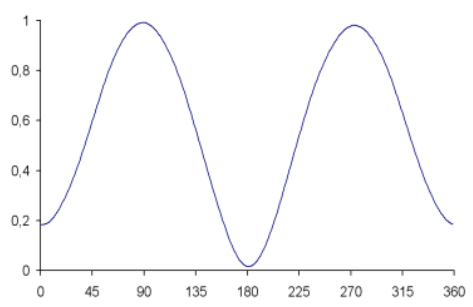

b)

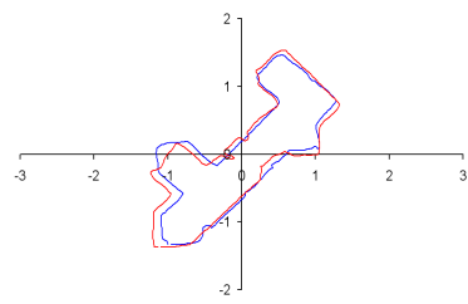

c)

Figure 3. Fine comparison between a pair of objects: a) Original objects, b) Dissimilarity measurement $d\left(f_{1} f_{2}\right)$ for $\alpha \epsilon\left[0^{o}, 1^{o}, \ldots 359^{\circ}\right]$, and c) The overlapped contours after rotating $f_{2}$ the angle that minimises the distance $\left(180^{\circ}\right)$

\subsection{Object symmetries. Point Group}

The fine comparison method described above is used to compute the PG of an object but, in this case, to compare each object to itself the following aspects have been considered:

- The order of rotational symmetry $C n$ of an object $O_{l}$ with contour $f_{l}$ is the number of local minima (under a threshold) of $d\left(f_{1} f_{l}\right)$.

- The order of reflection symmetry $D n$ of an object $O_{l}$ with contour $f_{l}$ is the number of local minima (under a threshold) of $d^{R}\left(f_{1} f_{1}\right)$, where the angles of reflection are: $\beta_{i}=\left(\alpha_{i} / 2\right), i=0,1, \ldots n-1$, where $\alpha_{i}$ is each rotation angle which minimises $d^{R}\left(f_{1} f_{l}\right)$. These angles are equally spaced according to the order of the symmetry group: $\beta_{i}=\beta_{0}+i(180 / n), i=0,1, \ldots n-1$. 


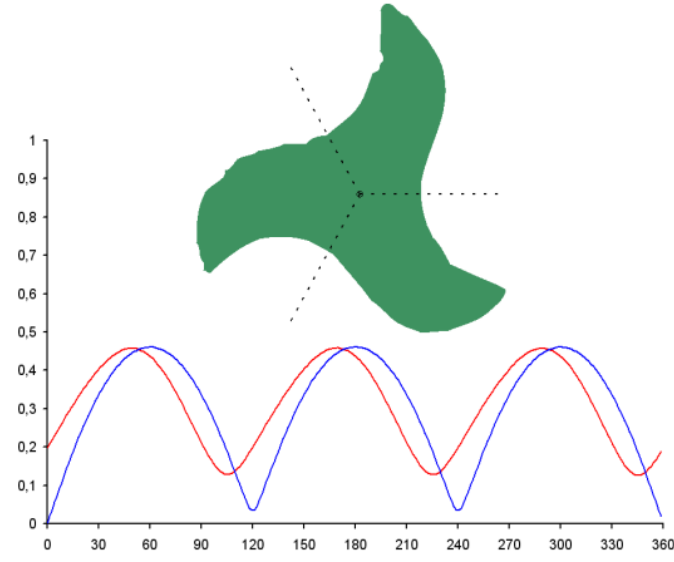

a)

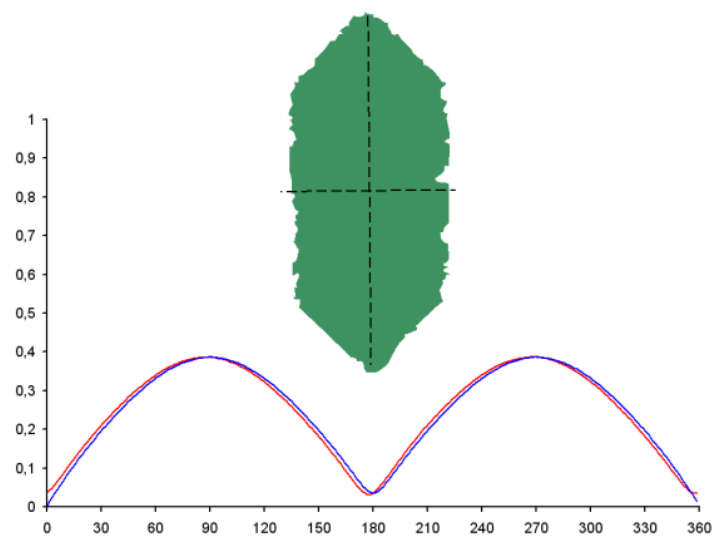

b)

Figure 4. Dissimilarity measurements $d\left(f_{1}, f_{1}\right)$ (blue) and $d^{R}\left(f_{1}, f_{1}\right)$ (red), $\alpha \in\left[0^{o}, 1^{o}, \ldots 359^{\circ}\right]$, for samples of Figure 1a and Figure $1 \mathrm{~b}$ respectively

The sample in Figure $4 \mathrm{a} d\left(f_{1}, f_{1}\right)$ has three minima below the threshold $(0.05)$, but the minima of $d^{R}\left(f_{1}, f_{1}\right)$ are always above the threshold. The minima are separated $120^{\circ}$, which means cyclic symmetry $C_{3}$. In Figure $4 \mathrm{~b}$ there are two local minima $\left(0^{\circ}\right.$ and $\left.180^{\circ}\right)$ below the threshold, which means dihedral symmetry $D_{2}$ with axes of $0^{\circ}$ and $90^{\circ}$.

\subsection{Description of objects}

For each object class, a representative object is randomly chosen as a relative reference, and the rotation and/or reflection of the other objects in the class with respect to it are then stored.

In the same way that an object with symmetry $C_{n}$ or symmetry $D_{n}$ with order $n$ has $n$ rotations (being invariant in all of them), there are $n$ rotations that relate the representative of the class with any other object in the class: $\alpha_{i}=\alpha_{0}+i(180 / n), i=0,1, \ldots n-1$.

The result after applying the operations included on the Object Level is a description of each object as a tuple $O_{k}=\left(R^{k}\right.$, colour $^{k}, p_{c}^{k}, A^{k}, \varphi_{\min }^{k}, I_{\min }^{k}, I_{\max }^{k}, C^{k}$, class $^{k}$, reflection $\left.^{k}, \alpha^{k}, p g^{k}, \beta^{k}\right)$, where the new features added are:

- $C^{k}$ : contour (closed sequence of 8-connected points).

- class $^{k}$ : class of the object.

- reflection ${ }^{k}$ : boolean value to indicate whether the object orientation is reflected with respect to the object selected as a reference in the class.

- $\alpha^{k}$ : orientation of the object with respect to the object selected as a reference in the class.

- $p g^{k}$ : order $n$ for Point Group (negative when $D_{n}$ and positive when $C_{n}$ ). 
- $\beta^{k}$ : angle of one reflection axis, whether the object has reflection symmetry.

276

277

278

279

280

281

282

283

284

285

286

\section{Structure Level}

This is the highest level, and its purpose is to obtain the Primitive Cell and Wallpaper Group. For each pair of objects of a class, there will be one or more isometries that relate them. If the same isometry is fulfilled for several pairs of objects, it is an isometry of the Wallpaper Group. The isometries can be the following:

\section{Rotation isometry}

For each pair of objects $O_{1}$ and $O_{2}$ belonging to the same class, with centroids $\left(x_{1}, y_{1}\right)$ and $\left(x_{2}, y_{2}\right)$, reflection ${ }^{1}=$ reflection $^{2}$ and $p g=C_{l}$ or $D_{1}$, there is a rotation of angle $\gamma=\alpha_{l^{-}} \alpha_{2}$, radius $r$ and centre $C$ which relate them, being:

$$
\begin{gathered}
r=\left|\frac{\sqrt{\left(x_{2}-x_{1}\right)^{2}+\left(y_{2}-y_{1}\right)^{2}} / 2}{\sin (\gamma / 2)}\right| \\
C\left\{\begin{array}{l}
\left(c_{x}-x_{1}\right)^{2}+\left(c_{y}-y_{1}\right)^{2}=r^{2} \\
\left(c_{x}-x_{2}\right)^{2}+\left(c_{y}-y_{2}\right)^{2}=r^{2}
\end{array}\right.
\end{gathered}
$$

where the true centre $C$ keeps the sign of the rotation angle, and when $\gamma=0^{\circ}$ and $r=\infty$, then the isometry is a translation. Thus, for two objects of the same class with $p g=C_{n}$ or $D_{n}$, the number of angles that relate both objects through rotation should be $n^{2}$, yet the angles are repeated, so there are $n$ rotations of angle $\gamma=\left(\alpha_{1}-\alpha_{2}\right)+l(360 / n)$, radius $r_{1}$ and centre $C_{1}$, which relate them (see Figure 5a). In the case of Figure 5b, $\gamma_{3}=0^{\circ}$ so there is a rotation of radius $r_{3}=\infty$ which corresponds to a translation.

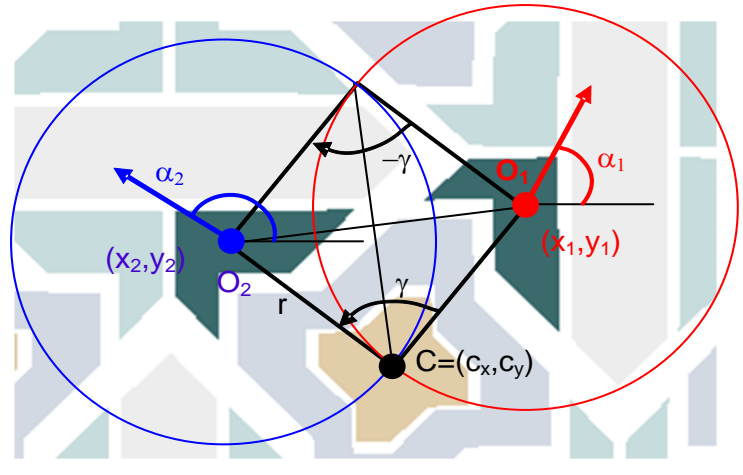

a)

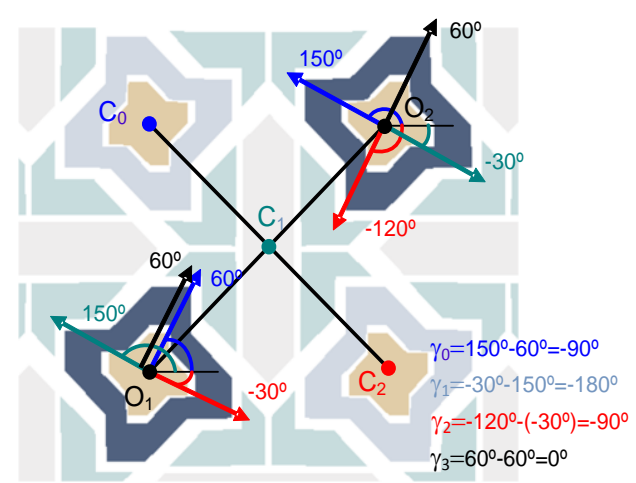

b)

Figure 5. Hand-drawn graphical information superimposed upon a WG sample to illustrate: a) Radius and rotation centres between two objects with $p g=C_{1}$, and b) Rotation centres between two objects with $p g=C_{4}$. Different colours have been use to represent centroids and orientations

For two objects $O_{1}$ and $O_{2}$ of the same class, with centroids $\left(x_{1}, y_{1}\right)$ and $\left(x_{2}, y_{2}\right)$, reflection ${ }^{l}=$ reflection $^{2}$ and 
$p g=C_{n}$ or $D_{n}$, there is a translation which relates them if there is a rotation angle $\gamma=\left(\alpha_{1^{-}} \alpha_{2}\right)+l(360 / n)=0^{\circ}$.

\begin{abstract}
The translation vector is the difference between the centroids of the objects: $D_{1-2}=\left(x_{1}-x_{2}, y_{1}-y_{2}\right)$.
\end{abstract}

\title{
Glide and non-glide reflection isometries
}

For two objects $O_{1}$ and $O_{2}$ of the same class, with centroids $\left(x_{1}, y_{1}\right)$ and $\left(x_{2}, y_{2}\right), p g=C_{n}$ and reflection ${ }^{1} \neq$ reflection $^{2}$, there are $n$ symmetry axes of angle $\delta_{1}$, a point $E$ (which is the midpoint between the centroids and is the same for all axes) and a glide glide $_{1}$ which relate them, being:

$$
\begin{aligned}
& \delta_{l}=\left(\alpha_{1}+\alpha_{2}\right) / 2+l(180 / n), l \epsilon[0, n-1] \\
& E=\left(e_{x}, e_{y}\right)=\left(\frac{x_{2}+x_{1}}{2}, \frac{y_{2}+y_{1}}{2}\right) \\
& \text { glide }_{l}=\left(x_{2}-x_{1}\right) \cdot \cos \delta_{1}+\left(y_{2}-y_{1}\right) \cdot \sin \delta_{1}
\end{aligned}
$$

where (6) are the projections of the vector between the centroids over the axis. If glide $_{1} \approx 0$ the symmetry axis is non-glide, otherwise it is a glide symmetry axis.

Thus, for two objects $O_{1}$ and $O_{2}$ of the same class, with centroids $\left(x_{1}, y_{1}\right)$ and $\left(x_{2}, y_{2}\right)$, with $p g=D_{n}$, there are $n$ symmetry axes of angle $\delta_{1}=\left(\beta_{1^{-}} \beta_{2}\right) / 2+l(180 / n)$, point $E$ and glide glide $e_{1}$ which relate them.

As happens with rotations, there are only $n$ values for $\delta_{l}$ that generate different angles, and therefore the number of symmetry axes is $n$. The two examples in Figure 6a and Figure $6 \mathrm{~b}$ show the symmetry axes between two pairs of objects of the same class with $\mathrm{pg}=C_{l}$ (calculated from object rotations, Figure 6a) and $p g=D_{l}$ (calculated from their internal symmetry axes, Figure 6b). On the other hand, Figure 7 shows the two symmetry axes between two objects of the same class with $p g=D_{2}$. Each symmetry axis is calculated from the combination of the internal axes represented with the same colour.

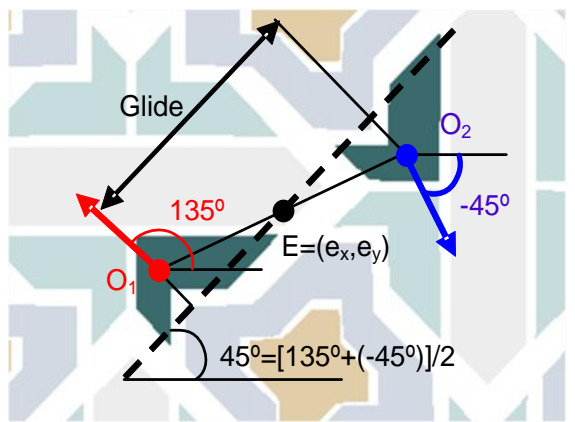

a)

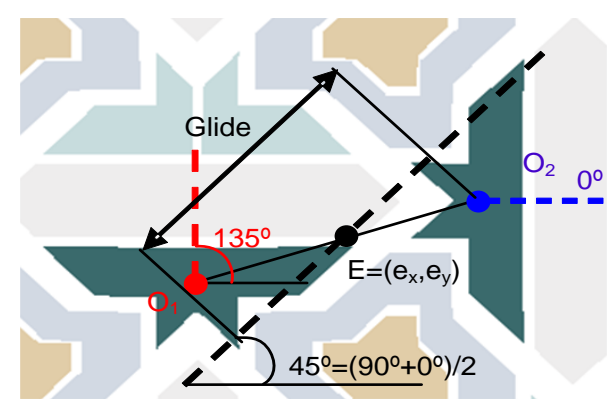

b)

Figure 6. Hand-drawn graphical information superimposed upon a WG sample to illustrate Glide reflection between two pairs of objects a) with $p g=C_{l}$, and b) $p g=D_{l}$ 


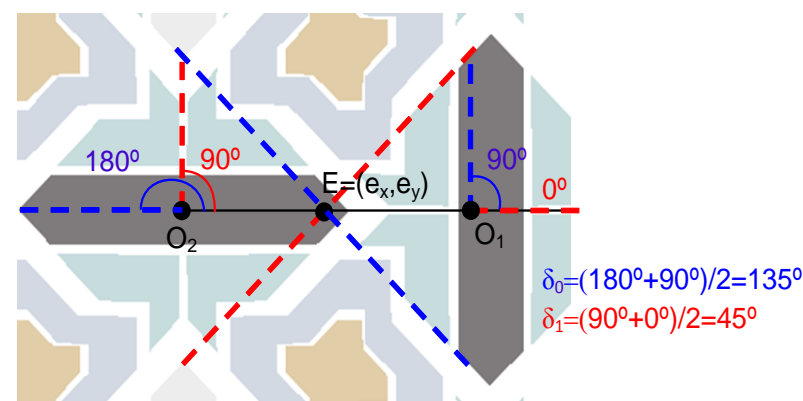

Figure 7. Hand-drawn graphical information superimposed upon a WG sample to illustrate Symmetry axes between two objects with $\mathrm{pg}=\mathrm{D}_{2}$

\subsection{Primitive Cell}

The lattice is defined by the translations in the pattern (see Figure 8) and thus, as defined in section 1.1, the $\mathrm{PC}$ is the smallest part of the pattern that is repeated by translations of the lattice.

Two approaches can be used to obtain the lattice: from Image Level operations and from a set of Scattered Elements. In the first case, some works have been carried out using techniques such as autocorrelation [39] [40], Markov Random Field (MRF) and Mean Shift Belief Propagation (MSBP) [28] to obtain the points forming the lattice, while others choose the directions which define the Primitive Cell using techniques such as Hough Transform [10]. If the lattice is obtained from a set of Scattered Elements, as for instance in [41] [42], some kind of visual similarity is performed in order to determine which elements are equal and then an optimisation of the existing translations is performed. This latest approach has been used in this work since it better suits the kind of objects found at the Object Level.

For each class of objects, the translation vectors between the objects with the same orientation are obtained. All vectors that are a linear combination of any pair of smaller vectors are then removed. Finally, only one pair of vectors remains $\left(L_{l}\right.$ and $\left.L_{2}\right)$ as the basis of a vectorial space defined by the positions of objects with the same rotation and reflection. Hence, the PC is defined by the vectors $L_{1}$ and $L_{2}$.

When more than one lattice is found, they are not independent so that the objects do not overlap. Therefore, the largest PC is chosen because it includes the others and it is the only one that is valid for the entire pattern. In Figure 8b the PC including the brown and green objects is the only one valid for the entire pattern and includes the other ones. 


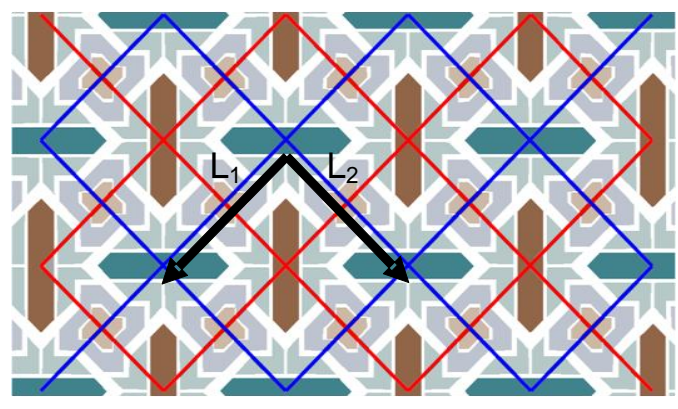

a)

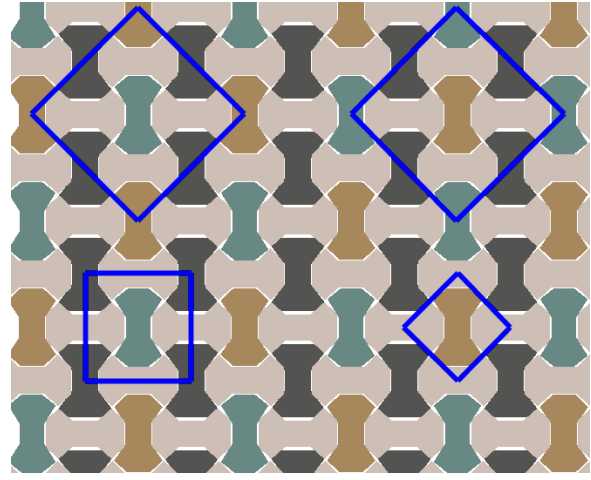

b)

Figure 8. Hand-drawn graphical information superimposed upon WG samples to illustrate: a) Sample of lattice among objects of a class with two different orientations represented with different colours and PC, and b) Sample of different PC belonging to different lattices for different objects

\subsection{Wallpaper Group}

Table 1 shows the diagram of the 17 Wallpaper Groups that exist together with their isometries and the classic nomenclature as well as the shape of their more generic PC [3]. The complexity of the isometries increases from left to right (order of rotation centres) and from top to bottom (presence of more symmetry axes). The solid black line of PC is not represented if it matches the symmetry axes, which are represented using a blue dashed line for non-glide axes and a red dashed line for glide axes and rotation centres (order 2: circumference, order 3: triangle, order 4: square, order 6: hexagon). 


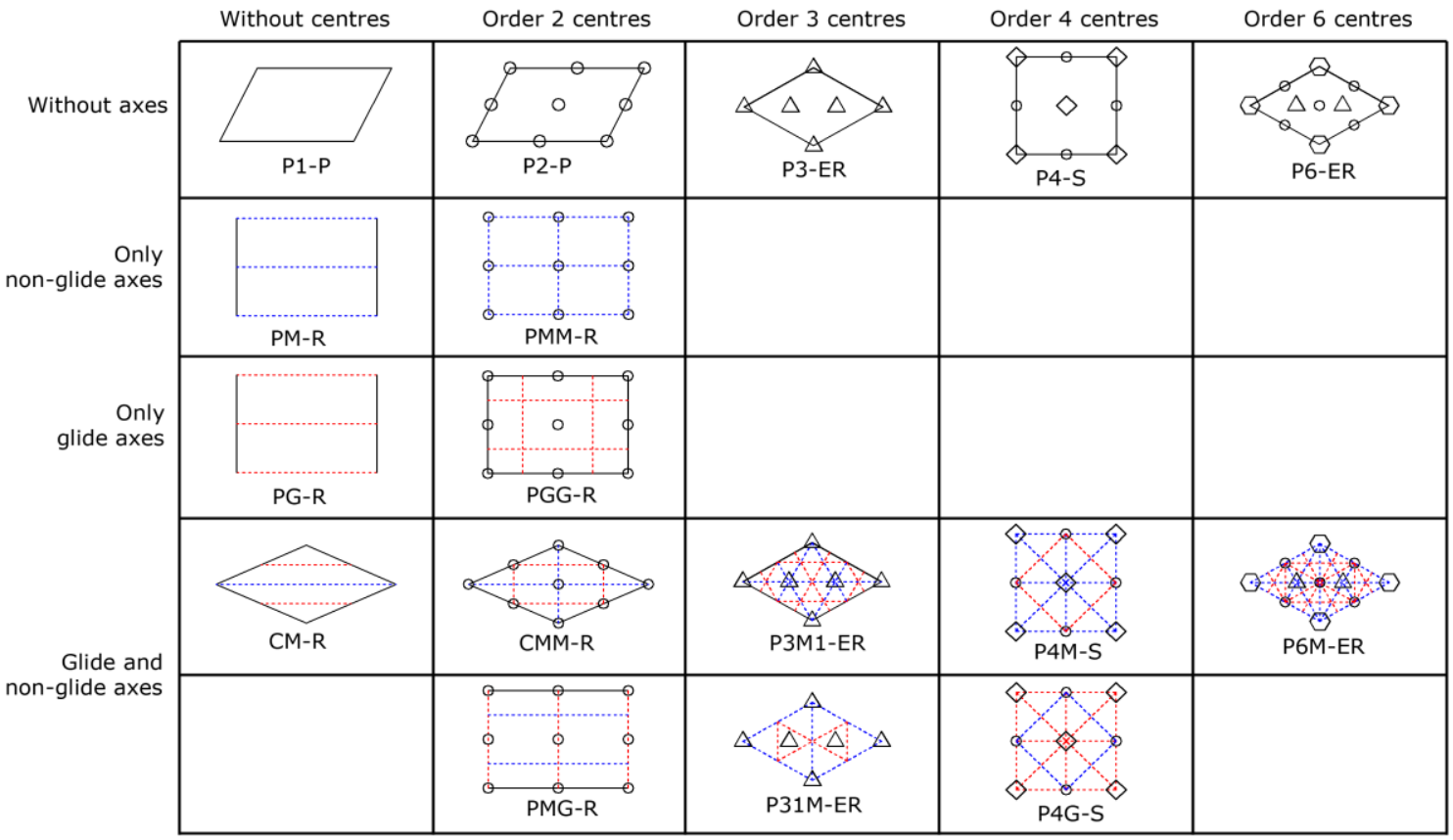

Table 1 shows three characteristics (Primitive Cell, Rotation Centres and Symmetry axes) that allow the WG to be detected for each class of objects in a pattern. These three characteristics and how to obtain them are explained in the next three subsections.

\subsubsection{Shape of Primitive Cell}

The shape of a PC may be a generic parallelogram, rectangle, square, rhombus or equilateral rhombus. The type of shape discards some Wallpaper Groups (e.g. if the PC is a generic parallelogram, the Wallpaper Group can only be a P1 or P2). This shape is obtained from translations by comparing the size and the angles of the sides of the PC (Figure 9). Due to the hierarchy among them, a WG may take the shape indicated, and all those derived more restrictively.

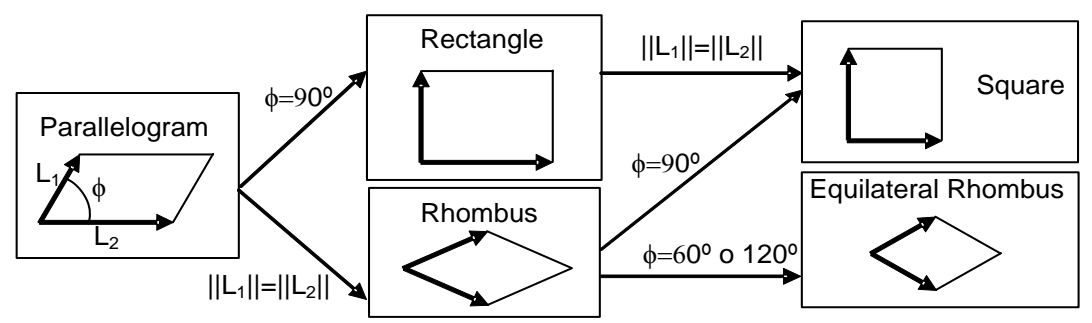

Figure 9. Hierarchy and features of each PC shape

\subsubsection{Order of rotation centres}

Each rotation centre is obtained from the rotations between objects of the same class, by matching their centres. The main feature is the order $\left|360^{\circ} / \gamma_{\min }\right|, \gamma_{\min }$ being the smaller rotation angle $\gamma_{l}$.

\subsubsection{Orientation of symmetry axes}


Each symmetry axis is obtained from the reflections, between objects of the same class, with the same angle $\delta_{l}$ and aligned; the symmetry axis glide is the lowest from all the symmetry axes between pairs of objects (if glide $\approx 0$, the symmetry axis is non-glide). The main features are:

- If the angle $\delta_{l}$ is parallel or orthogonal to the sides and diagonals of PC (see Figure 9).

- If it is a glide symmetry axis or non-glide symmetry axis.

As each object class can generate a different PC, it can generate different rotation centres and symmetry axes and, consequently, different WG. Therefore, the WG for each object class is obtained separately, and the least restrictive one is chosen, i.e. the one whose isometries are present in the others, which is the only one valid for the entire pattern. In the pattern shown in Figure 10, WG P4 is the least restrictive one, its rotation centres are present in P4M (Figure 10a), but the symmetry axes of P4M are not included in P4 (Figure 10b), so P4 is the only one that is valid for the entire pattern.

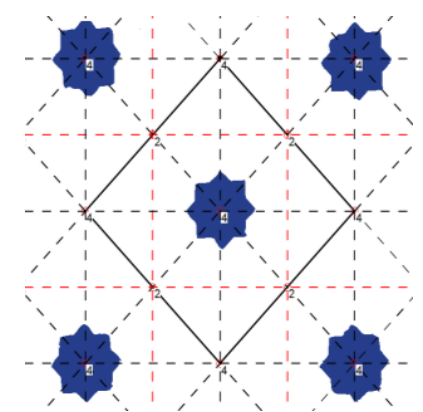

a)

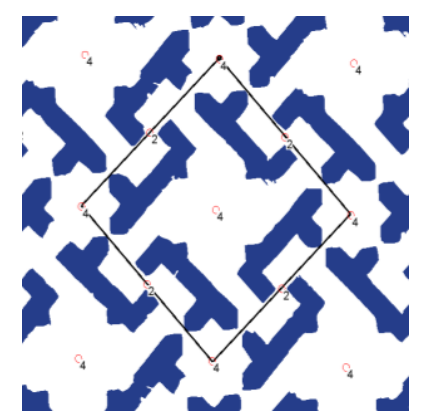

b)

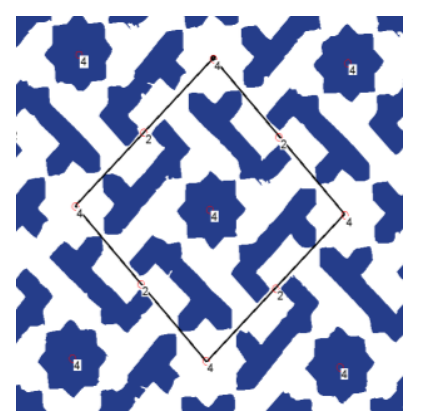

c)

Figure 10. Output of the application for a pattern sample with two WG: a) WG P4M for the stars; and b) WG P4 for the objects of the other class; c) WG P4 is chosen for the entire pattern

\subsection{Description of the structure}

The pattern structure contains all the isometries, and it is contained in a tuple $E=\left(O, V_{1}, V_{2}, P C, W P, \theta\right)$, where:

- $O$ : origin of Primitive Cell.

- $V_{1}, V_{2}$ : vectors of Primitive Cell sides.

- $P C$ : shape of Primitive Cell (generic parallelogram, rectangle, square, rhombus, equilateral rhombus).

- WP: Wallpaper Group (P1, PM, PG, CM, P2, PMM, PMG, PGG, CMM, P3, P31M, P3M1, P4, P4M, P4G, P6, P6M).

- $\theta$ : angle of an axis (with respect to PC elements: sides or diagonals) for Wallpaper Groups PM, PG, $\mathrm{CM}$ and PMG. This is necessary because in the PM and PG groups the symmetry axes may be 

parallel to one side or another, in the CM group the symmetry axes may be parallel to one diagonal or another, and in the PMG group non-glide symmetry axes may be parallel to one side or another.

\section{Results and Discussion}

Figure 11 shows the output of the application for a sample with a synthetic pattern. The pattern has different orders of rotation centres and different symmetry axes, which have allowed the accuracy of the method developed to be validated. Figure 11a shows the pattern Wallpaper Group, which is P4G (with a PC Square). Figure 11b shows the objects obtained by applying the proposed method with the different orientations in which they appear in the pattern, containing their internal symmetries and Point Group. Figure 12 shows a screenshot of the interface of the application developed. The different stages of the method levels and a message box with the information obtained about the WG for the entire pattern are shown on the left.

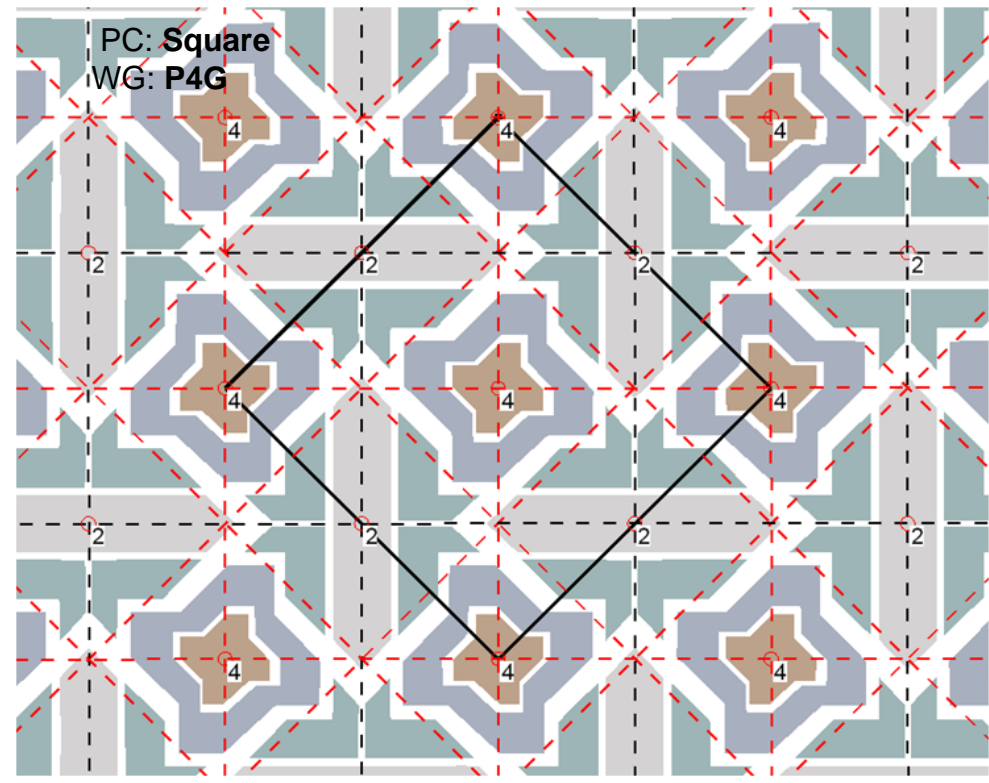

a)
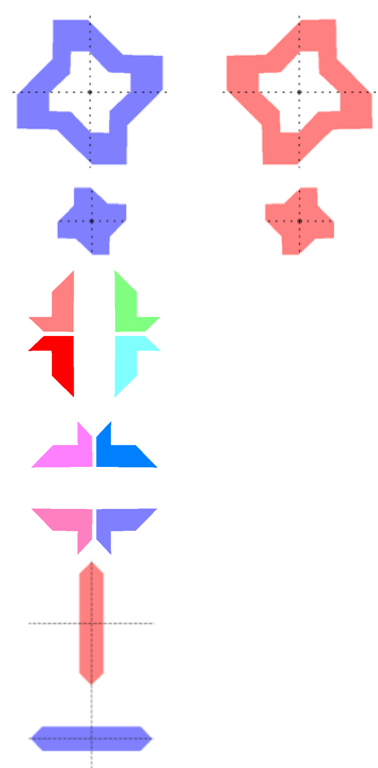

PG: C4

2 orientations

PG: C4

2 orientations

PG: C1

8 orientations

PG: D2

2 orientations
402

Figure 11. Output of the application for a sample with a synthetic pattern: a) Synthetic pattern analysis with its structure, and b) The object classes contained (different colour for the different orientations for each class) 


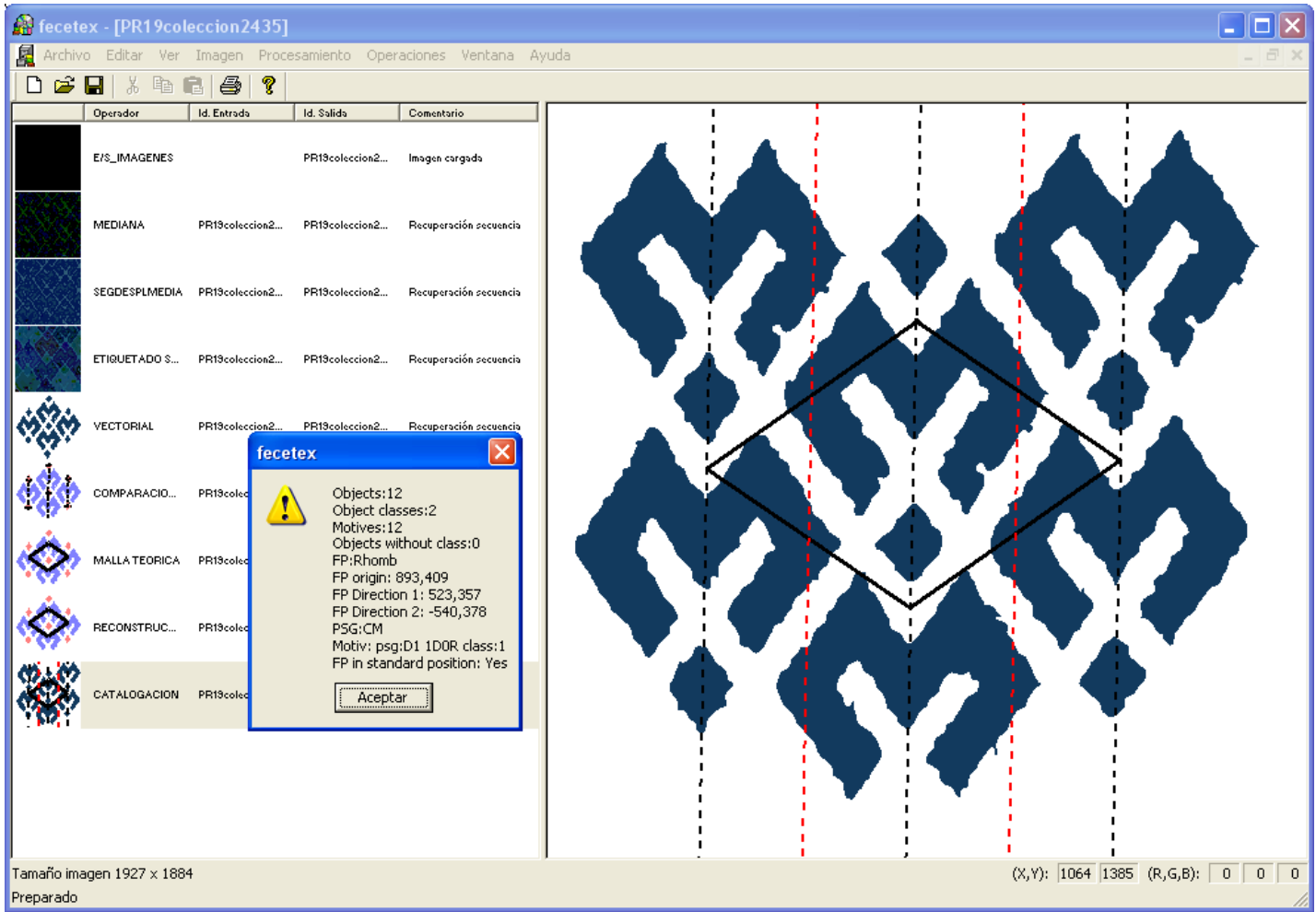

Figure 12. Interface of the application software that was developed

\subsection{Results on IGP}

The proposed method was tested over a set of 96 images containing Islamic Geometric Patterns from the Alhambra of Granada (Spain) (42), the Alcázar of Seville (Spain) (23), Morocco (26, mostly from [43]), and the Great Mosque of Damascus (Syria) (5).

Figure 13 to Figure 15 show the analysis of three real IGP with the application and its output. Figure 13 and Figure 14 show the object classes found with their PG and the different orientations in which the objects appear. Particularly, for the IGP in Figure 13 the analysis found a different WG when considering only the shape of objects and discarding their colour. In the case of Figure 14 the analysis found the WG considering only the stars, which has more isometries than the global WG. In the case of the IGP in Figure 15, only two colours were considered for the foreground and for the background, WG P3 being found as the valid WG for the entire pattern. These three examples show the flexibility provided by the Object Level and why, in most cases, the WG perceived does not always coincide with the WG of the pattern, as [43] pointed out. Compared to the analysis from the methods presented in the state of the art, none of them can obtain information about objects like shapes, classes, orientations and Point Groups (rotational and reflection symmetry). For instance, for the sample in Figure 13 the pattern analysis would be performed always taking into account the colour of the objects, so the WG in Figure 13d would never be found. For 
the sample in Figure 14 the pattern analysis would only obtain the solution in Figure 14c.

424

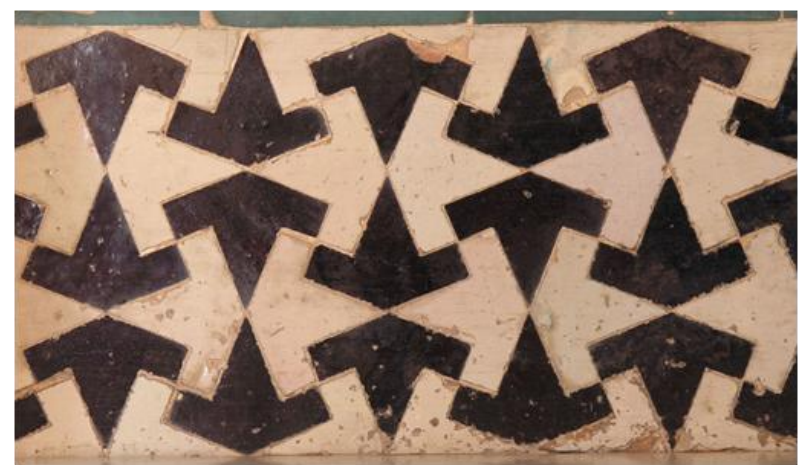

a)

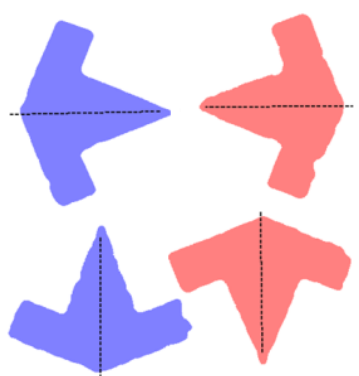

b)
PG: D1

2 orientations

PG: D1

2 orientations

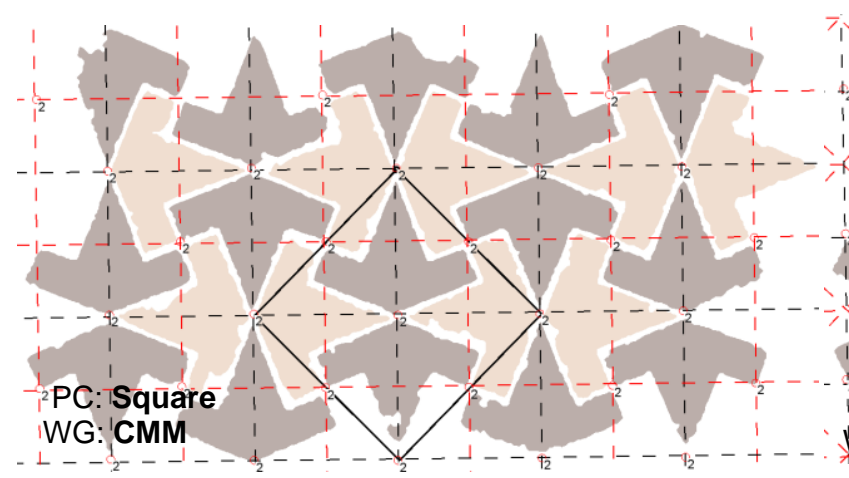

c)

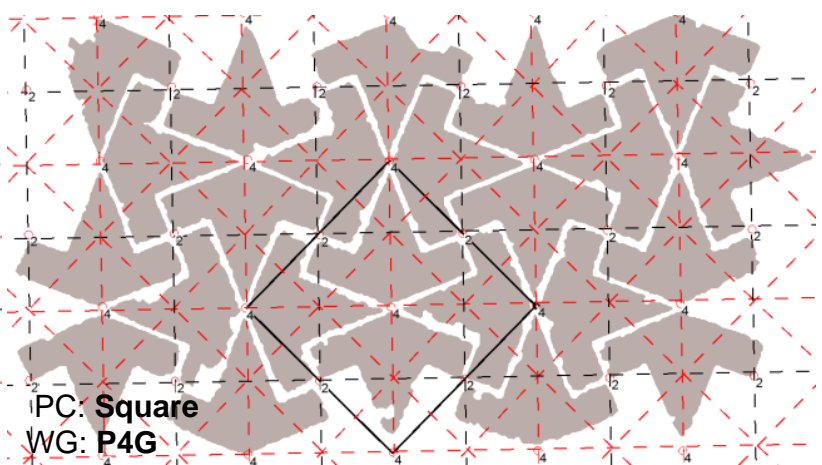

d)

Figure 13. a) Islamic Geometric Pattern from the Alhambra of Granada; b) Object classes; c) WG CMM after analysis; and d) WG P4G taking into account only the shape of the objects found 


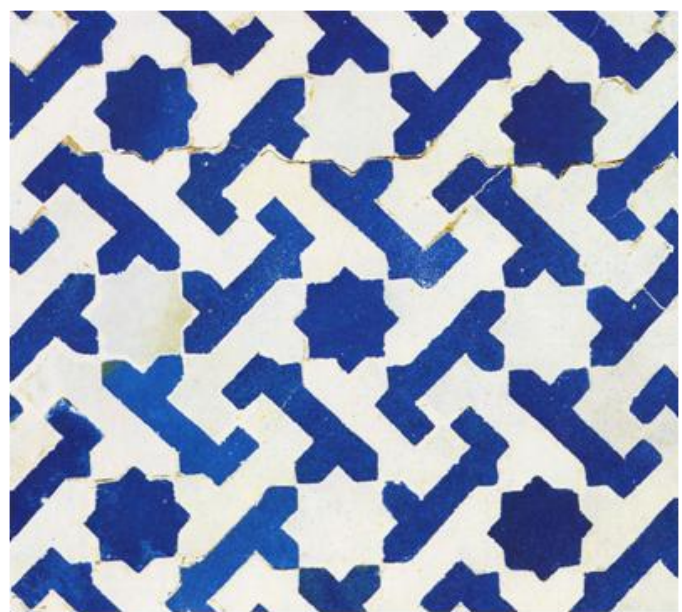

a)

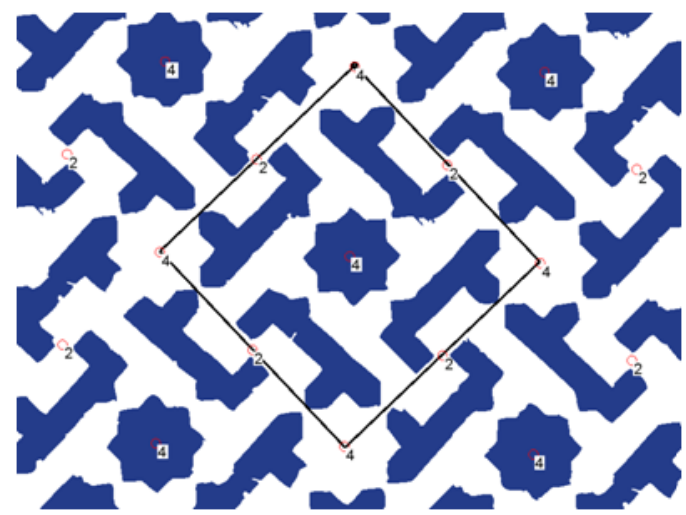

c)

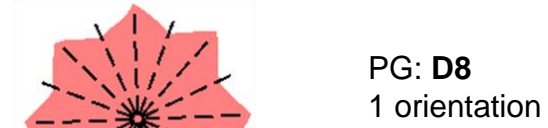

1 orientation

PG: C1

4 orientations

Figure 14. a) Islamic Geometric Pattern from the Palais Royal of Rabat (Morocco); b) Object classes; c) WG P4M found only for stars; and d) WG P4 found for the entire pattern (the stars and other objects)

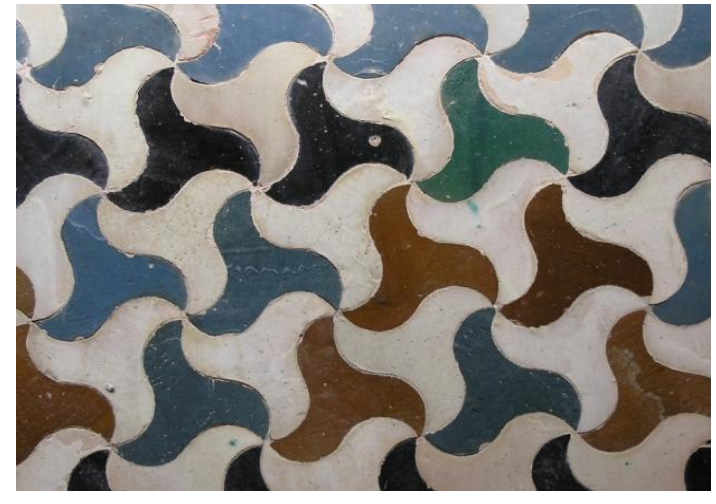

a)

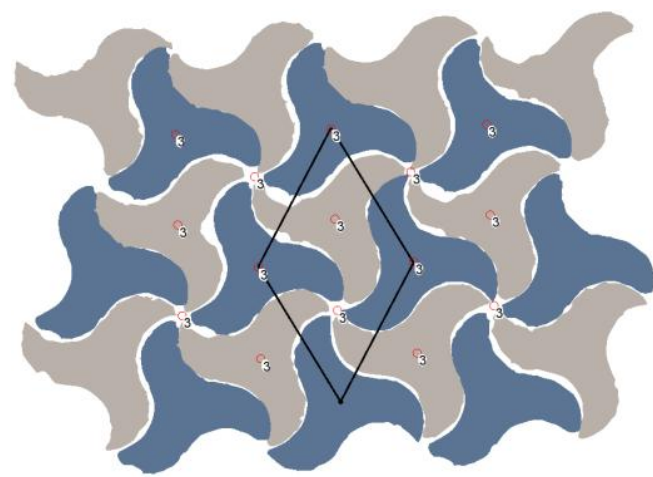

PC: Equilateral Rhombus WG: P3

b)

Figure 15. a) Islamic Geometric Pattern from the Alhambra of Granada; and b) WG P3 found for the entire 
Figure 16 shows the percentage of Wallpaper Groups and Point Groups found in the IGP analysed. There are only five patterns from the Great Mosque of Damascus, so they are included in the total score. The WG which appear most often (P4M, P4, CMM, P6M, P6, P3 and P4G) have dense structures, plenty of isometries, and the existence of WG for only a particular object class, usually CMM combined with PMM or $\mathrm{P} 4 \mathrm{G}$, is also very common. In contrast, some groups have few occurrences or never appear (PG, PMG, PGG, P31M, P1, PM, P2, P3M1). It is also noteworthy that the predominant WG are different (P4M, CMM and P4 in the Alhambra, P4 in the Alcázar, and P4M and P6M in Morocco).

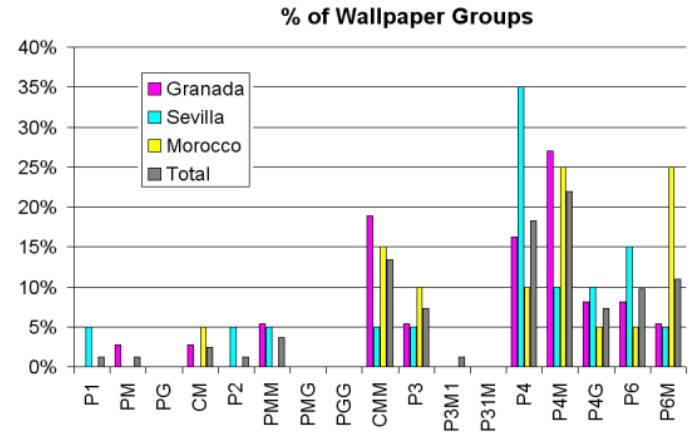

a)

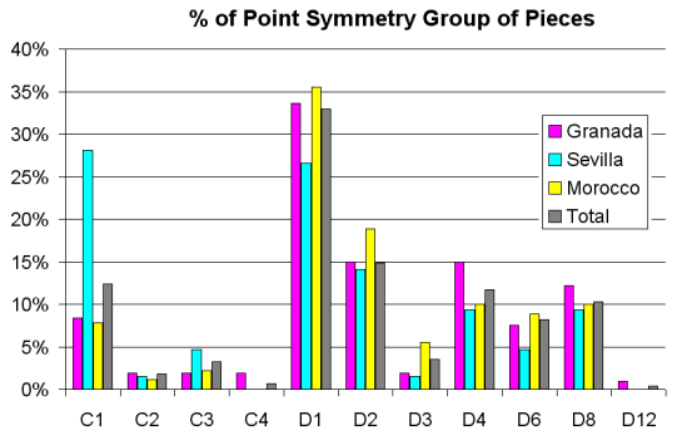

b)

Figure 16. a) Percentage of Wallpaper Groups found in the IGP analysed; and b) Percentage of Point Groups found in the pieces of IGP analysed

Figure 17a shows the results of the correct identification of the WG in the IGP coming from different locations. The WG was found for $85 \%$ of the patterns, while for $73 \%$ pieces of all classes, patterns were also found with their Point Group successfully obtained. The patterns from the Alcázar of Seville are more complex and contain a larger number of different pieces, and therefore the success rate was lower when obtaining the pieces, although the identification of the WG was still good.

Figure $17 \mathrm{~b}$ shows the results of the correct identification of the different WG. Dismissing the case of PMM (of which there are only 5 patterns) the worst results are obtained for P4, because most of them are patterns from the Alcázar of Seville, which are more complex and with a much wider range of different pieces. 


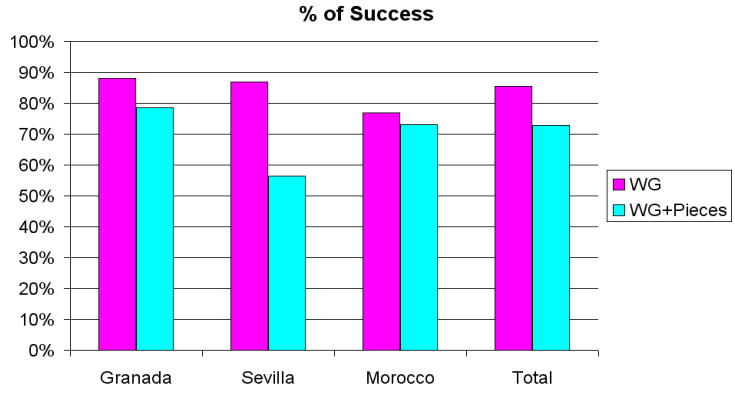

a)

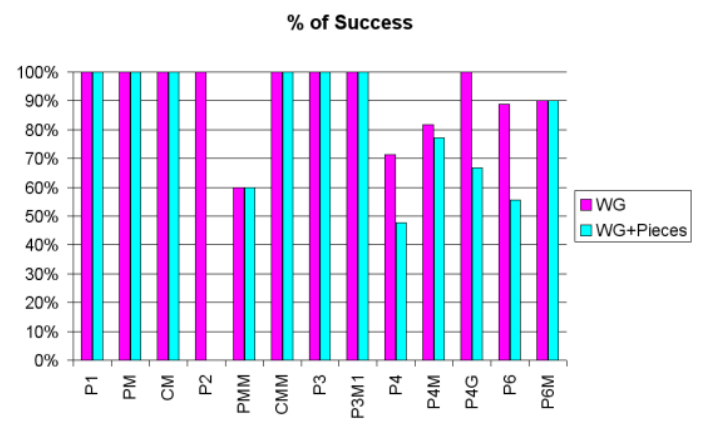

b)

Figure 17. Success rate in the analysis carried out for the IGP: a) Success by location: two different rates are shown, i.e. the patterns with the WG found successfully, and the patterns where all classes of pieces were found, and b) Success rate by $\mathrm{WG}$

In most of the cases where the application failed, this was due to a low performance of the segmentation process (Image Level) caused by the poor quality of the patterns, since they belong to ancient damaged patterns (Figure 18 left). The problems at the Image Level severely influenced the outcome of the further levels, for example, by causing errors in the comparison of objects and in obtaining their symmetries.

Even in the cases where segmentation was good, another source of problems that was encountered came from the inaccuracies introduced by the handmade nature of the different pieces: colour was not uniform, the objects of the same class were not exactly equal, the shapes were not perfectly symmetrical and the geometric transformations which relate objects of the same class were not exactly equal. This caused mistakes when determining the morphological features and the relations among objects. Finally, some patterns could not be analysed because of the high complexity and large number of different pieces they contained, as shown in Figure 18 middle and right.
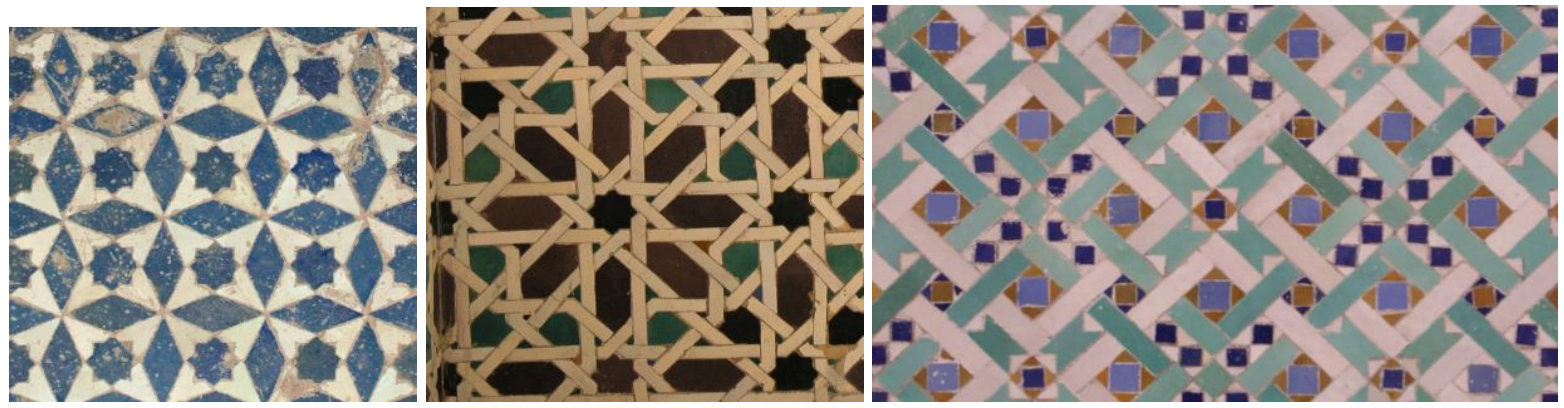

Figure 18. Images of some examples of IGP that failed

\subsection{Parameters, computational cost and intermediate results of the algorithm}

The table 2 shows the stages of the algorithm, their parameters and their computational cost. In the last column it is shown the elapsed time in each stage for the sample of Islamic Geometric Pattern of Fig. 13. In 
this sample, the full image size is $2000 \times 1500$ and the full pattern has 33 objects. The analysis was performed with an Intel Core 2 Duo $3 \mathrm{Ghz}$ and the time of each stage includes the generation of the image.

Table 2. Stages of the algorithm with their corresponding parameters, their computational cost and the elapsed time of each stage for a specific sample

\begin{tabular}{|c|c|c|c|}
\hline STAGES & PARAMETERS & $\begin{array}{l}\text { COMPUTATIONAL } \\
\text { COST }\end{array}$ & $\begin{aligned} \text { TIME* } \\
\text { (sec) }\end{aligned}$ \\
\hline $\begin{array}{l}\text { Pre-process } \\
\text { (Gaussian blur) }\end{array}$ & Radius: size of Gaussian kernel & O(pixels) & 3.88 \\
\hline $\begin{array}{l}\text { Segmentation } \\
\text { (Watershed) }\end{array}$ & $\begin{array}{l}\text { MinEdgeHeight: minimum difference of grey level between an edge and } \\
\text { a neighbouring valley, if the difference is lower, it is not an edge and the } \\
\text { neighbouring valleys join } \\
\text { MaxValleyHeight: maximum grey level of a valley, if the level is higher, it } \\
\text { is not a valley, it is considered background }\end{array}$ & O(pixels) & 8.94 \\
\hline $\begin{array}{l}\text { Morphological } \\
\text { operations }\end{array}$ & Iterations & O(objects) & 7.69 \\
\hline Contour extraction & --- & O(objects) & 11.78 \\
\hline $\begin{array}{l}\text { Object } \\
\text { classification }\end{array}$ & $\begin{array}{l}\text { MaxCoarse: maximum area ratio and axis ratio } \\
\text { MaxFine: maximum contour distance }\end{array}$ & O(objects ${ }^{2}$ ) & 2.14 \\
\hline Object symmetries & MaxFine: maximum contour distance & O(objects) & 0.81 \\
\hline Primitive Cell & $\begin{array}{l}\text { SizeTolerance: maximum size difference between vectors to be } \\
\text { considered equal } \\
\text { AngleTolerance: maximum angle difference between vectors to be } \\
\text { considered equal } \\
\text { MinPercentage: minimum ratio of occurrences of a vector with respect } \\
\text { to the number of objects of its class and orientation }\end{array}$ & $O\left(\right.$ objects $\left.^{2}\right)$ & 0.25 \\
\hline Wallpaper Group & $\begin{array}{l}\text { DistanceTolerance (\% of PC lower side): maximum difference between } \\
\text { glides or distance between axis or distance between rotation centres to } \\
\text { be considered equal } \\
\text { AngleTolerance: maximum angle difference between axis to be } \\
\text { considered equal } \\
\text { MinPercentage: minimum ratio of occurrences of an axis or rotation } \\
\text { centre with respect to the number of objects of its class }\end{array}$ & O(objects $\left.{ }^{2}\right)$ & 0.63 \\
\hline
\end{tabular}

* Time consumed at each stage for the IGP of the Fig. 13

Fig. 19 shows the intermediate graphical results of each stage of the algorithm for the Islamic Geometric 


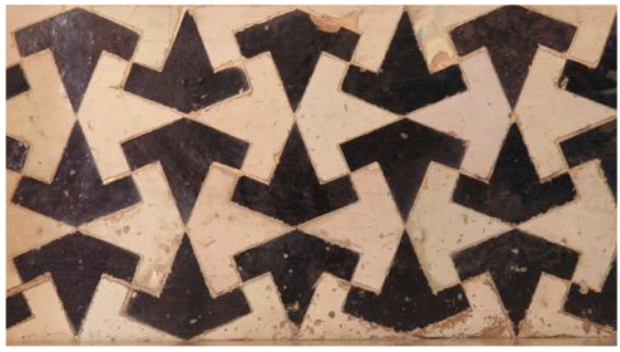

a)

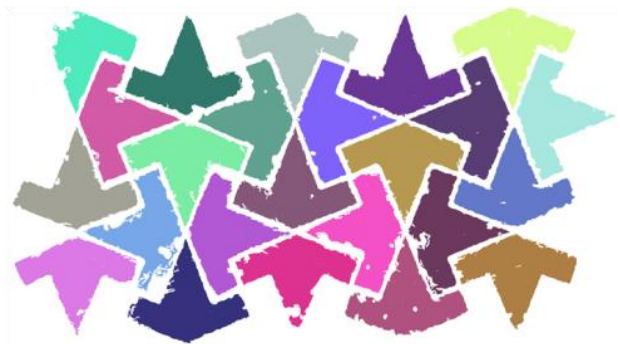

c)

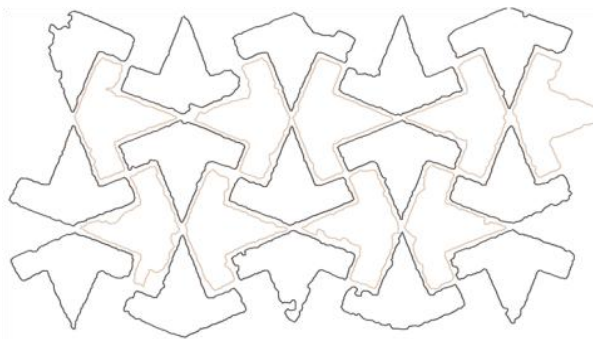

e)

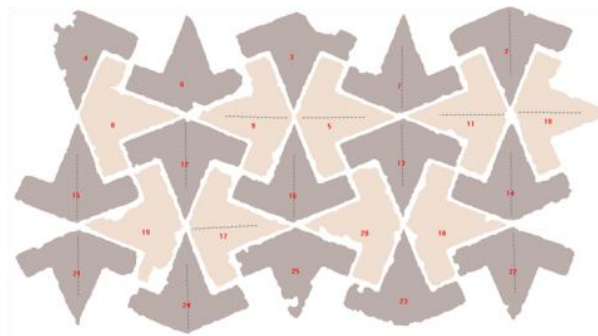

g)

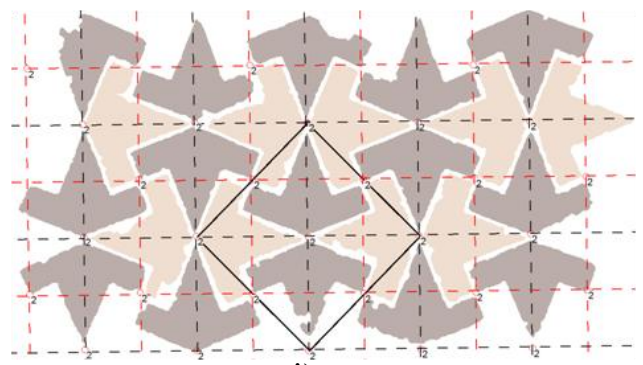

i)

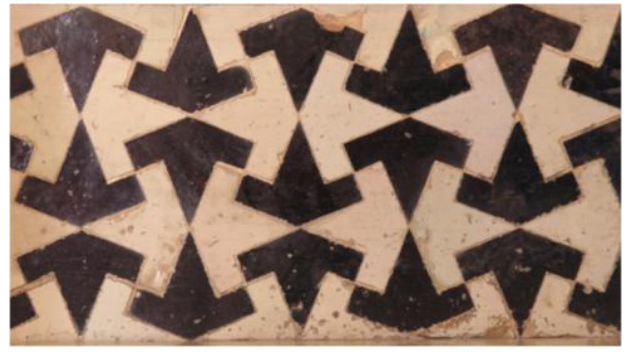

b)

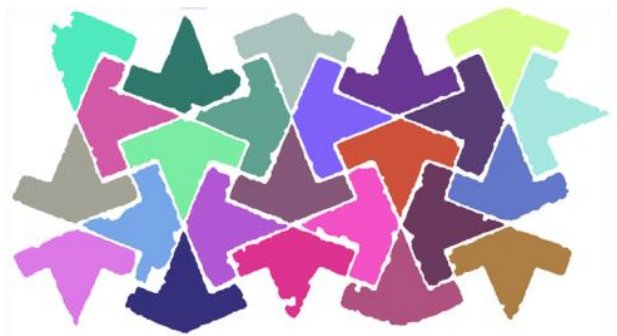

d)

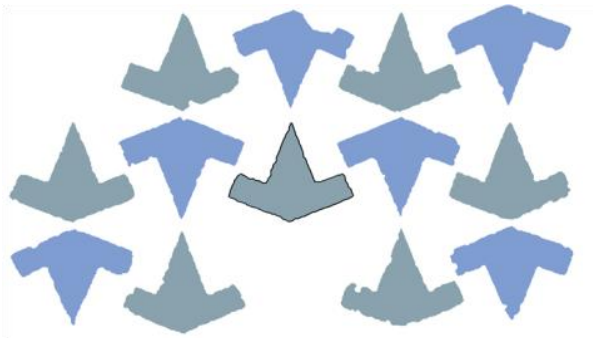

f)

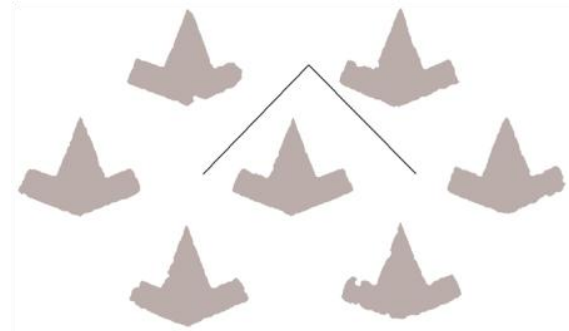

h)

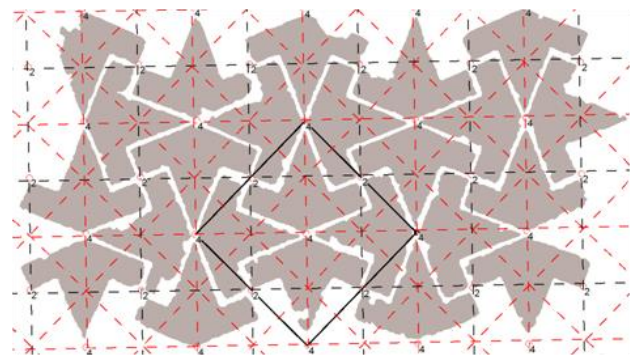

j)

Figure 19. Intermediate results of each stage for the IGP of Fig. 13: a) Original image; b) Pre-process stage

(Gaussian filter); c) Segmentation stage (Watershed); d) Morphological Operations stage (Closing); e) 
Contour Extraction stage; f) Object Classification stage; g) Object Symmetries stage; h) Primitive Cell stage; i) Wallpaper Group stage; and j) Wallpaper Group stage discarding colour

Describing Fig. 19 briefly, Fig. 19a shows the original image; Fig. 19b is the image after a Gaussian filter; Fig. 19c is the segmented image where each object is represented with a random colour; Fig. 19d shows the image after closing operations where each object is represented with a random colour; Fig. 19e shows the contours extracted; Fig. 19f shows only one class of objects found, where the objects with the same orientation are represented with the same colour and the representative object is outlined; Fig. $19 \mathrm{~g}$ shows each object represented with its Point Group; Fig. 19h shows the pair of vectors remaining between objects with the same orientation of one class (two black lines); Fig. 19i shows the Wallpaper Group resulting a WG CMM that has been obtained taking into account the colour of the objects; and finally, Fig. $19 \mathrm{j}$ shows the Wallpaper Group resulting a WG P4M that has been obtained discarding the colour of the objects.

\subsection{Application of the method to alternative datasets}

In order to test the flexibility of the proposed method, some images containing mosaics from alternative datasets have been analysed. Figure 20 shows some examples of WG from the Wikipedia (http://en.wikipedia.org/wiki/Wallpaper_group) and Figure 21 shows some examples of paving slabs from public constructions and fabrics [23]. Table 2 shows the results of the application for all the mosaics from the Wikipedia that were analysed.

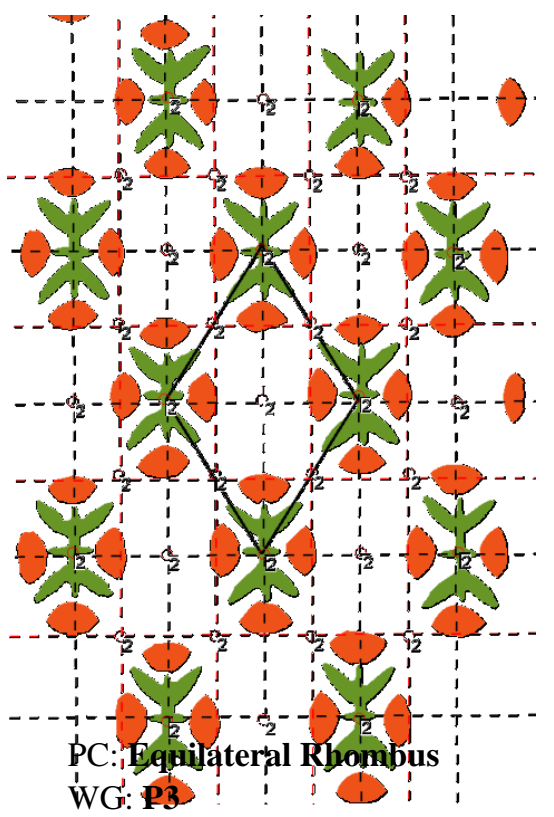

a)

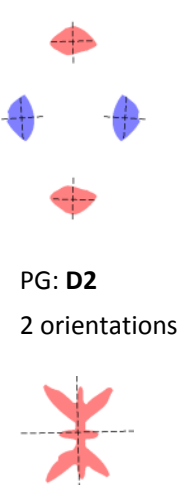

PG: D2 1 orientation

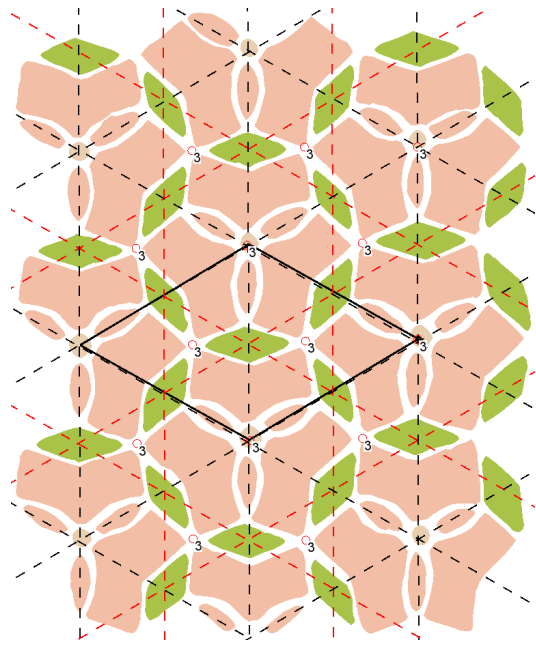

PC: Equilateral Rhombus WG: P31M

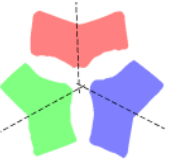

PG: D1 3 orientations ix

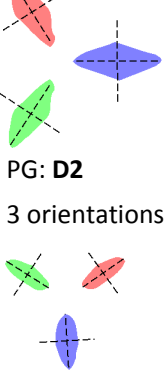

PG: D2 3 orientations

b) 


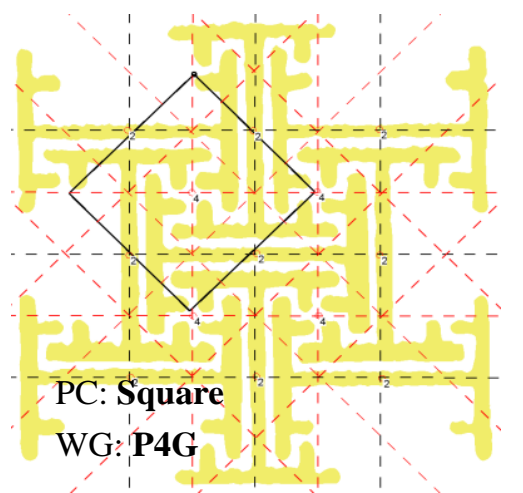

c)

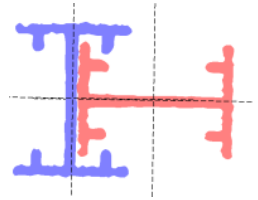

PG: D2

2 orientations

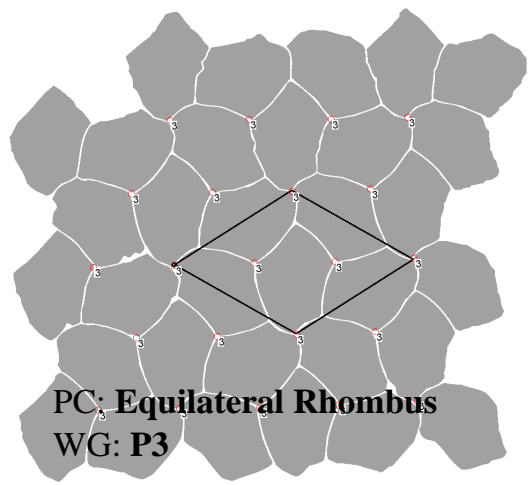

d)
502

503

505

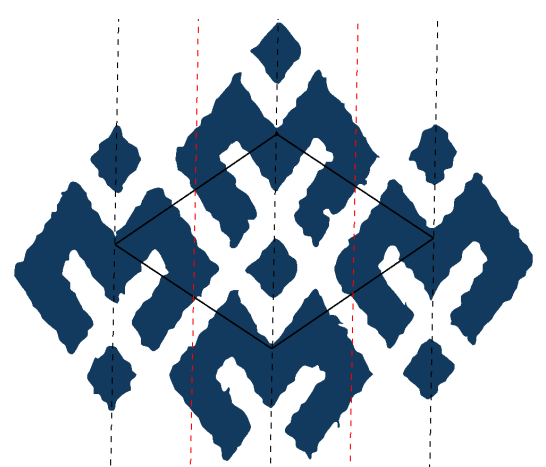

PC: Rhombus

WG: CM

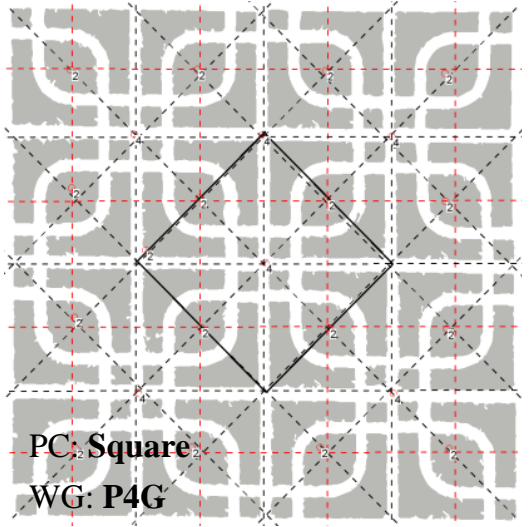

a)

WG: P4G; and d) WG: P3

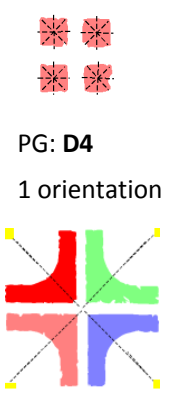

PG: D1

4 orientations
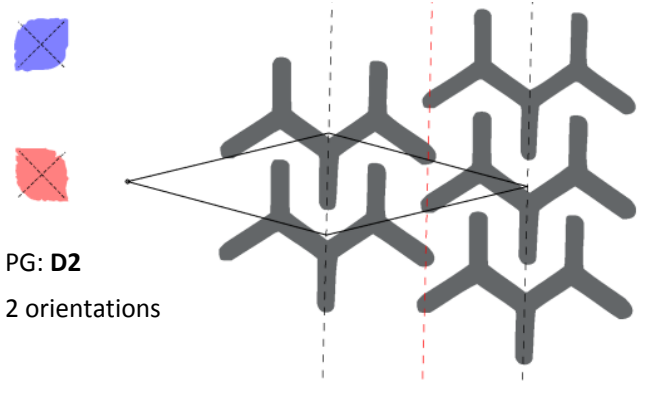

PC: Rhombus

WG: $\mathbf{C M}$

b)

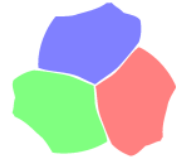

PG: C1

3 orientations

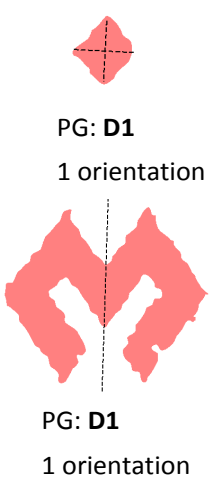

c)

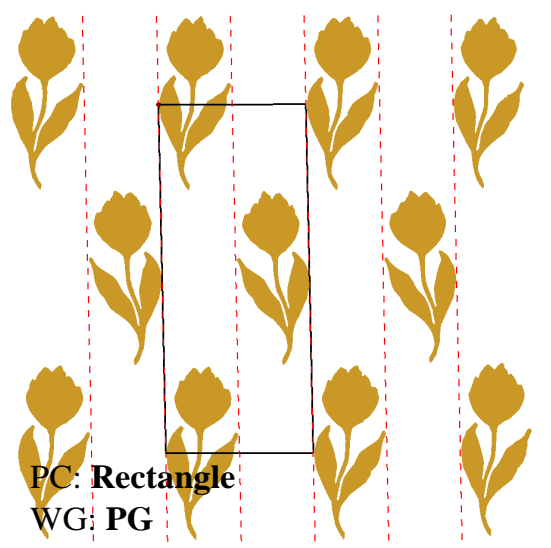

d) 


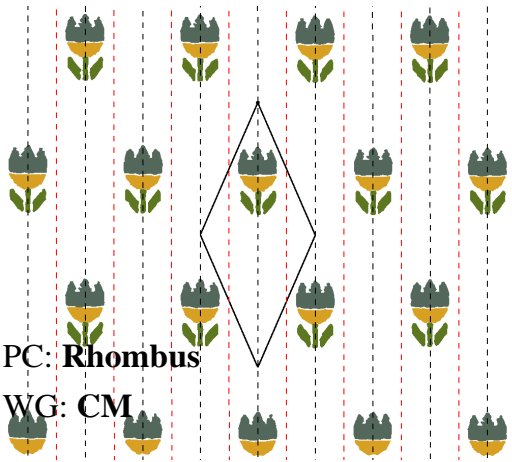

wid cm $\circlearrowleft \omega$

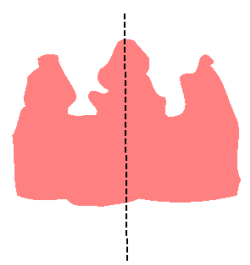

PG: D1

1 orientation

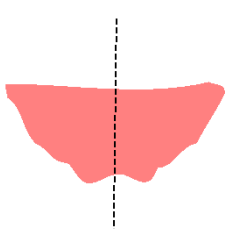

PG: D1

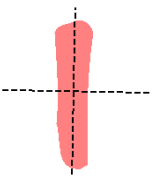

PG: D2

1 orientation

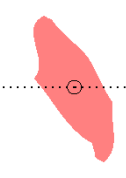

PG: C2

2 orientations

e)

Figure 21. Application output for some examples of paving slabs from public constructions and fabrics.

Table 3. Results for all the samples from the Wikipedia that were analysed

\begin{tabular}{|c|c|c|c|c|}
\hline Name & $\begin{array}{l}\text { Fig. in this } \\
\text { article }\end{array}$ & $\begin{array}{c}\text { WG } \\
\text { (Other WG) }\end{array}$ & $\begin{array}{c}\text { Objects } \\
\text { Point Group } \\
\text { (Number of classes) } \\
\end{array}$ & Comments \\
\hline $\begin{array}{l}\text { Wallpaper_group-cm-6 } \\
\text { (Persian tapestry).jpg }\end{array}$ & & & & WG not found \\
\hline $\begin{array}{l}\text { Wallpaper_group-cm-7 (Indian metal- } \\
\text { work at the Great Exhibition in 1851).jpg }\end{array}$ & & $\mathrm{CM}$ & $\begin{array}{l}\text { C1 (2) } \\
\text { D1 (3) } \\
\text { D2 (2) }\end{array}$ & $\begin{array}{l}\text { Some object class } \\
\text { not found }\end{array}$ \\
\hline $\begin{array}{l}\text { Wallpaper_group-cmm-4 } \\
\text { (Persian tapestry).jpg }\end{array}$ & Fig.20a & CMM & D2 (2) & \\
\hline $\begin{array}{l}\text { Wallpaper_group-p3-1 (Street pavement in } \\
\text { Zakopane, Poland).jpg }\end{array}$ & Fig. 20d & P3 & C1 (1) & \\
\hline $\begin{array}{l}\text { Wallpaper_group-p3m1-1 (Persian glazed } \\
\text { tile (ignoring colours p6m)).jpg }\end{array}$ & & & & WG not found \\
\hline $\begin{array}{l}\text { Wallpaper_group-p4-2 } \\
\text { (Ceiling of Egyptian tomb).jpg }\end{array}$ & & $\begin{array}{c}\text { P4 } \\
\text { (P4M all object } \\
\text { classes except } 1 \text { ) }\end{array}$ & $\begin{array}{l}\text { C2 (1) } \\
\text { D1 (1) } \\
\text { D2 (1) }\end{array}$ & $\begin{array}{c}\text { Some object class } \\
\text { not found }\end{array}$ \\
\hline $\begin{array}{l}\text { Wallpaper_group-p4g-1 } \\
\text { (Bathroom linoleum, U.S.).jpg }\end{array}$ & & P4G & D2 (1) & \\
\hline $\begin{array}{l}\text { Wallpaper_group-p4g-2 } \\
\text { (Painted porcelain, China).jpg }\end{array}$ & Fig. 20c & P4G & D2 (1) & \\
\hline $\begin{array}{l}\text { Wallpaper_group-p4m-1 (Ornamental } \\
\text { painting, Nineveh, Assyria).jpg }\end{array}$ & & P4M & $\begin{array}{l}\text { D2 (1) } \\
\text { D4 (4) }\end{array}$ & \\
\hline $\begin{array}{l}\text { Wallpaper_group-p4m-4 } \\
\text { (Egyptian tomb).jpg }\end{array}$ & & & & WG not found \\
\hline $\begin{array}{l}\text { Wallpaper_group-p4m-6 } \\
\text { (Persian glazed tile).jpg }\end{array}$ & & P4M & $\begin{array}{l}\text { D1 (3) } \\
\text { D4 (1) }\end{array}$ & \\
\hline $\begin{array}{l}\text { Wallpaper_group-p31m-1 } \\
\text { (Persian glazed tile).jpg }\end{array}$ & Fig. 20b & P31M & $\begin{array}{c}\text { D1 (1) } \\
\text { D2 (2) } \\
\text { Dinf (circle) (1) } \\
\end{array}$ & \\
\hline $\begin{array}{l}\text { Wallpaper_group-p31m-2 } \\
\text { (Painted porcelain, China).jpg }\end{array}$ & & P31M & D3 (2) & \\
\hline $\begin{array}{l}\text { Wallpaper_group-p31m-3 } \\
\text { (Painting, China).jpg }\end{array}$ & & P31M & D3 (3) & \\
\hline Wallpaper_group-pg-2 (Pavement with & & & & WG not found \\
\hline
\end{tabular}




\begin{tabular}{|c|c|c|c|}
\hline herringbone pattern in Salzburg).jpg & & & \\
\hline $\begin{array}{l}\text { Wallpaper_group-pgg-2 } \\
\text { (Pavement in Budapest, Hungary).jpg }\end{array}$ & PGG & D2 (1) & \\
\hline $\begin{array}{l}\text { Wallpaper_group-pm-3 (Dress of a figure } \\
\text { in a tomb at Biban el Moluk, Egypt).jpg }\end{array}$ & $\begin{array}{c}\text { PM } \\
\text { (CM ignoring } \\
\text { colours) }\end{array}$ & D1 (2) & \\
\hline $\begin{array}{l}\text { Wallpaper_group-pm-5 (Indian } \\
\text { metalwork at the Great Exhibition } \\
\text { in 1851. This is almost pm (ignoring } \\
\text { short diagonal lines between ovals } \\
\text { motifs, which make it p1)).jpg }\end{array}$ & & & WG not found \\
\hline $\begin{array}{l}\text { Wallpaper_group-pmm-2 (Mummy } \\
\text { case stored in The Louvre).jpg }\end{array}$ & $\begin{array}{c}\text { PMM } \\
\text { (P4M ignoring } \\
\text { colours) }\end{array}$ & $\begin{array}{c}\text { D2 (1) } \\
\text { D4 (2) } \\
\text { Dinf (circle) (1) }\end{array}$ & \\
\hline
\end{tabular}

For the method developed to work properly, the contours of the objects must be well defined and closed, and the size should be large enough to be considered objects. However, we can see that the method has achieved good results and in some cases has even obtained other WG ignoring colours or ignoring some object classes.

All the images used in this article can be downloaded from http://personales.upv.es/maalbor/FilesWG/IGP.rar and http://personales.upv.es/maalbor/Files-WG/Wiki-Others.rar

\section{Conclusions and Further Work}

This work presents a method to gain an understanding of IGP patterns (and any kind of pattern presenting a Wallpaper Group) based on Image Processing, Pattern Recognition and Symmetry Groups, the main novelty of which is the addition of a new object-oriented level of knowledge. This method is structured on three main levels of knowledge: the lower level or Image Level, the intermediate level or Object Level, and the upper level or Structure Level.

The Image Level is pixel-oriented and its aim is to obtain the regions of interest in the image of the pattern, these regions constitute the objects that form the pattern. The Object Level is a higher level from which the features of the objects are extracted (including their Point Group), a classification of the objects is carried out, and the relationships among them are determined, which introduces a deeper analysis of the patterns and hence a deeper understanding can be found. Other important advantages introduced by the Object Level are the obtaining of the WG of each object class, apart from the WG of the entire pattern, and also the possibility of analysing the pattern taking into account the objects found regardless of their colour (considering only their shape). Finally, on the Structure Level, the pattern structure (Primitive Cell and Wallpaper Group) is obtained from the relationships, that is, the isometries (translations, symmetry axes and rotations) among objects of the same class, provided by all the understanding attained on the Object 
Level.

The main contribution of this work resides precisely in these two levels. None of the methods presented in the state of the art is able to obtain this information.

The tests performed using a set of 96 Islamic Geometric Patterns show that this method is capable of obtaining Wallpaper Groups and all classes of pieces with their corresponding Point Group from different types of real IGP. In addition, the tests show differences between Wallpaper Group patterns and Point Group pieces, depending on their origin, as shown in Figure 16a. Moreover, the method has been tested on some images from alternative datasets (Wikipedia, paving slabs from public constructions and fabrics) and was seen to be capable of achieving the expected results in most of the samples analysed.

Possible applications of this computational method include pattern classification, cataloguing of ceramic coatings, creating databases of decorative patterns, creating pattern designs, pattern comparison between different cultures, tile cataloguing, and so on.

Plans have been made to carry out further work in order to:

- Use the information obtained to 'reconstruct' the pattern geometrically, that is, to retrieve missing objects, to make objects of the same class look exactly alike, to perform the geometric transformations which relate objects of the same class in exactly the same way, and to make the shapes perfectly symmetrical. This reconstruction can be performed by copying objects and applying geometric transformations of the Wallpaper Group (to recover missing objects of the same class), redoing the shape of objects according to their point group (making the shapes perfectly symmetrical), adjusting their position and orientation to the translations, centres of rotation and symmetry axes of the pattern, and any other key feature.

- Processing complex patterns successfully, which can be done, for instance, by grouping objects and also by classifying groups to obtain the isometries of the pattern from groups instead of objects.

\section{Acknowledgments}

The authors wish to thank the Patronato de la Alhambra y Generalife (Granada, Spain) and the Patronato del Real Alcázar de Sevilla (Seville, Spain) for their valuable collaboration in this research work.

\section{References}

[1] S. E. Palmer, Vision Science. Photons to Phenomenology, MIT Press, 2002. 
[2] P.B. Yale, Geometry and Symmetry: Holen-Day, 1968.

[3] D. Schattschneider, "The Plane Symmetry Groups: Their Recognition and Notation", The American Mathematical Monthly, vol. 85, no. 6, pp. 439-450, Jun/Jul 1978.

[4] B. Grünbaum and G.C. Shephard, Tilings and Patterns: W.H. Freeman and Company, 1987.

[5] H. Alexander, "The Computer/Ploter and the 17 Ornamental Design Types", Proc. 2nd Conf. Computer Graphics and Interactive Techniques (SIGGRAPH '75), pp. 160-177, 1975.

[6] J. Weeks, "Programs that Can Automatically Generate 2D Planar Crystallographic Patterns. Kali", http://www.geom.umn.edu/apps/Kali/, 1995.

[7] [Terrazo] (Xaos Tools) http://photoshop.pluginsworld.com/plugins_86/adobe/photoshop/xaos_tools/terrazzo.html

[8] [Symmetry Works] (Artlandia) http://www.artlandia.com/products/SymmetryWorks

[9] V. Ostromoukhov, "Mathematical Tools for Computer-Generated Ornamental Patterns", Lecture Notes in Computer Science, vol. 1375, pp. 192-223, 1998, doi: 10.1007/BFb0053272

[10] Y. Liu, R.T Collins and Y. Tsin, “A Computational Model for Periodic Pattern Perception Based on Frieze and Wallpaper Groups", IEEE Trans. Pattern Analysis and Machine Intelligence, vol. 26, no. 3, pp. 354-371, 2004, doi:10.1109/TPAMI.2004.1262332

[11] V. Asha, P. Nagabhushan and N.U. Bhajantri, "Automatic Extraction of Texture-Periodicity using Superposition of Distance Matching Functions and their Forward Differences", Pattern Recognition Letters, vol. 33, no. 5, pp. 629-640, April 2012, doi:10.1016/j.patrec.2011.11.027.

[12] M. Agustí, J.M. Valiente and A. Rodas, "Lattice Extraction Based on Symmetry Analysis", Proc. 3rd Int. Conf. Computer Vision Theory and Applications (VISAPP'08), pp.396-402, 2008, ISBN: 978-989-8111-21-0.

[13] M. Agustí, A. Rodas, J.M. Valiente, "Classification of Repetitive Patterns Using Symmetry Group Prototypes", Proc. 5th Iberian Conference on Pattern Recognition and Image Analysis (IbPRIA'11), pp. 84-91, June 2011, doi: 10.1007/978-3-642-21257-4_11

[14]H.Y.T. Ngana, G.K.H. Panga and N.H.C. Yung, "Motif-based Defect Detection for Patterned Fabric", Pattern Recognition, vol. 41, no. 6, pp. 1878-1894, June 2008, doi:10.1016/j.patcog.2007.11.014.

[15] M.O. Djibril and R.O.H. Thami, "Islamic Geometrical Patterns Indexing and Classification using Discrete Symmetry Groups", ACM J. Computing and Cultural Heritage, vol. 1, no. 2, article 10, October 2008, doi:10.1145/1434763.1434767.

[16] A.M. Aljamali, "Classification and Design of Islamic Geometric Patterns Using Computer Graphics", Proc. 2nd Int. Conf. Visualisation (VIZ’09), pp. 253-258, Jun/Jul 2009, doi:10.1109/VIZ.2009.46.

[17] P. Rasouli, A, Bastanfard, A. Rezvanian and O. Jalilian, "Fast Algorithms for Computer Generated Islamic Patterns of 8-ZOHREH and 8-SILI", Proc. 9th Pacific Rim Conf. on Multimedia (PCM'08), pp. 825-829, Dec. 2008, doi:10.1007/978-3-540-89796-5_91.

[18] I. Jowers, M. Prats and H. Eissa, "A Study of Emergence in the Generation of Islamic Geometric Patterns", Proc. 15th Int. Conf. Computer-Aided Architectural Design Research in Asia (CAADRIA'10), pp. 39-48, 2010.

[19] A. Aljamali and E. Banissi, "Grid Method Classification of Islamic Geometric Patterns”, Geometric Modelling, M. Sarfraz, ed., pp. 234-254, Kluwer Academic Publishers, 2004.

[20] M.O. Djibril and R.O.H. Thami, "A new Quadtree-Based Symmetry Transform (QST) with Application to ArabAndalusian Images Indexing", Proc. 2nd Int. Symp. on Comunications, Control and Signal Processing (ISCCSP'06), pp. 13-15, March 2006.

[21] M. Valor Valor, F. Albert Gil, J.M. Gomis Martí and M., "Textile and Tile Pattern Design Cataloguing Using Automatic Detection of the Plane Symmetry Group", Proc. Computer Graphics International (CGI'03), pp. 112119, Jul. 2003, doi:10.1109/CGI.2003.1214455. 
[22] J.M. Valiente González, F. Albert Gil, M. Carretero Rocamora and J.M. Gomis Martí, "Structural Description of Textile and Tile Pattern Designs Using Image Processing", Proc. 17th Int. Conf. on Pattern Recognition (ICPR'04), pp. 498-503, Aug. 2004, doi:10.1109/ICPR.2004.1334175.

[23] G. Medioni, M-S. Lee and C-K. Tang, A Computational Framework for Segmentation and Grouping: Elsevier Science, 2000.

[24]D.G.Fernández-Pacheco, F.Albert, N.Aleixos, J.Conesa, M.Contero, "Automated tuning of parameters for the segmentation of freehand sketches", Proceedings of the International Conference on Computer Graphics Theory and Applications (GRAP2011), pp.321-329, 2011.

[25] K.N. Plataniotis and A.N. Venetsanopuolos, Color Image Processing and Applications: Springer-Verlag, 2000.

[26] M. Sonka, V. Hlavac and R. Boyle, Image Processing, Analysis, and Machine Vision: Thomson Learning, 2007.

[27]D. Comaniciu and P. Meer, "Robust Analysis of Feature Spaces: Color Image Segmentation", Proc. 1997 Conference on Computer Vision and Pattern Recognition (CVPR'97), pp.750-755, Jun. 1997, doi:10.1109/CVPR.1997.609410.

[28] M. Park, K. Brocklehurst, R.T. Collins and Y. Liu, "Deformed Lattice Detection in Real-World Images Using Mean-Shift Belief Propagation”, IEEE Trans. Pattern Analysis and Machine Intelligence, vol. 31, no. 10, pp. 1804-1816, 2009, doi:10.1109/TPAMI.2009.73.

[29] S. Beucher and C. Lantuejoul, "Use of Watersheds in Contour Detection", Proc. Int. Workshop on Image Processing, Real-time Edge and Motion Detection/estimation, Rennes, France, Sept. 1979.

[30] J. Serra, Image Analysis and Mathematical Morphology: Academic Press, Inc., 1983.

[31] M.K. Hu, "Visual Recognition by Moment Invariants", IRE Trans. Information Theory, vol. 8, no. 2, pp.179-187, Feb. 1962, doi: 10.1109/TIT.1962.1057692

[32] A.K. Jain, Fundamentals of Digital Image Processing: Prentice-Hall, Inc., 1989.

[33] G.E. Martin, Transformation Geometry: An Introduction to Symmetry: Springer-Verlag, 1982.

[34]Z. Falomir, LL. Museros, L., González-Abril, M.T., Escrig and J.A. Ortega, "A Model for the Qualitative Description of Images Based on Visual and Spatial Features", Computer Vision and Image Understanding, vol. 116, no. 6, pp. 698-714, June 2012, doi:10.1016/j.cviu.2012.01.007.

[35]Z. Falomir, LL. Museros, L. González-Abril and F. Velasco, "Measures of Similarity between Qualitative Descriptions of Shape, Colour and Size applied to Mosaic Assembling", Journal of Visual Communication and Image Representation, vol. 24, no. 3, pp. 388-396, April 2013, doi:10.1016/j.jvcir.2013.01.013.

[36]D. Geiger, T.L. Liu and R.V. Kohn, "Representation and Self-similarity of Shapes", IEEE Trans. Pattern Analysis and Machine Intelligence, vol. 25, no. 1, pp. 86-99, Jan. 2004, doi:10.1109/TPAMI.2003.1159948.

[37]P.J. Otterloo, A Contour-Oriented Approach to Shape Analysis: Prentice-Hall International, 1991.

[38] R.W. Conners and C.A. Harlow, "Toward a Structural Textural Analyzer Based on Statistical Methods", Computer Graphics and Image Processing, vol. 12, no. 3, pp. 224-256, March 1980, doi:10.1016/0146664X(80)90013-1.

[39]H.C. Lin, L.L. Wang and S.N. Yang, "Extracting Periodicity of a Regular Texture Based on Autocorrelation Functions", Pattern Recognition Letters, vol. 18, no. 5, pp. 433-443, May. 1997, doi:10.1016/S01678655(97)00030-5

[40]T. Leung and J. Malik, "Detecting, Localizing and Grouping Repeated Scene Elements", Proc. 4th European Conf. Computer Vision (ECCV'96), pp. 546-555, April 1996, doi:10.1007/BFb0015565.

[41]F. Schaffalitzky, and A. Zisserman, "Geometric Grouping of Repeated Elements within Images", Shape, Contour, and Grouping in Computer Vision, D.A. Forsyth, V. Di Gesu, J.L. Mundy, and R. Cipolla, eds., pp. 165-181, Springer-Verlag, 1999.

[42] A. Paccard, Le Maroc et l'Artisanat Traditionnel Islamique dans l'Arquitechture: Editions Ateliers 74, 1981. 
[43] A.D.F. Clarke, P.R. Green, F. Halley and M.J. Chantler, "Similar Symmetries: The Role of Wallpaper Groups in Perceptual Texture Similarity", Symmetry, vol. 3, pp. 246-264, 2011, doi:10.3390/3020246. 